\title{
DYNAMICS OF A THREE SPECIES RATIO-DEPENDENT FOOD CHAIN MODEL WITH DIFFUSION AND DOUBLE FREE BOUNDARIES*
}

\author{
DAWEI ZhANG ${ }^{1}$, BeIPING DUAN ${ }^{2}$ AND BinXIANG DAI ${ }^{3, * *}$
}

\begin{abstract}
This paper focuses on the dynamics of a three species ratio-dependent food chain model with diffusion and double free boundaries in one dimensional space, in which the free boundaries represent expanding fronts of top predator species. The existence, uniqueness and estimates of the global solution are discussed firstly. Then we prove a spreading-vanishing dichotomy, specifically, the top predator species either successfully spreads to the entire space as time $t$ goes to infinity and survives in the new environment, or fails to establish and dies out in the long run. The long time behavior of the three species and criteria for spreading and vanishing are also obtained. Besides, our simulations illustrate the impacts of initial occupying area and expanding capability on the dynamics of top predator for free boundaries.
\end{abstract}

Mathematics Subject Classification. 35K51, 35R35, 92B05, 35B40.

Received November 14, 2019. Accepted July 29, 2020.

\section{INTRODUCTION}

In ecosystems, Prey-predator interaction is one of the basic interspecies relations, and it is also the basic block of more complicated food chain, food web, and biophysical network structures (e.g. [16]). A general food chain model is described by the system of differential equations

$$
\left\{\begin{array}{l}
\widetilde{N}_{t}=\widetilde{N} \varphi(\widetilde{N})-\frac{1}{e_{1}} \rho_{1}(\widetilde{N}, \widetilde{P}) \widetilde{N} \\
\widetilde{P}_{t}=\rho_{1}(\widetilde{N}, \widetilde{P}) \widetilde{P}-\tilde{m}_{1} \widetilde{P}-\frac{1}{e_{2}} \rho_{2}(\widetilde{P}, \widetilde{Z}) \widetilde{P} \\
\widetilde{Z}_{t}=\rho_{2}(\widetilde{P}, \widetilde{Z}) \widetilde{Z}-\tilde{m}_{2} \widetilde{Z},
\end{array}\right.
$$

where $\widetilde{N}, \widetilde{P}, \widetilde{Z}$ stand for the population density of prey, predator, and top predator, respectively (cf. [1, 4, 29]). $\rho_{1}(\widetilde{N}, \widetilde{P})$ and $\rho_{2}(\widetilde{P}, \widetilde{Z})$ are the functional response of predators; $e_{i}, i=1,2$ are the yield constants; $\tilde{m}_{i}, i=1,2$ are the normal death rates of predators. The function $\varphi(\widetilde{N})$ represents the per capita growth rate of $\widetilde{N}$. A

* This work was supported by the National Natural Science Foundation of China (Grant Nos. 11871475 and 11901091).

Keywords and phrases: Ratio-dependent food chain model, double free boundaries, spreading-vanishing dichotomy; long time behavior.

${ }^{1}$ School of Mathematics and Big Data, Foshan University, Foshan 528000, PR China.

2 Shenzhen JL Computational Science and Applied Research Institute, Shenzhen 570100, PR China.

3 School of Mathematics and Statistics, Central South University, Changsha 410083, PR China.

** Corresponding author: bxdai@csu.edu.cn 
TABLE 1. Parameters table of problem (1.2).

\begin{tabular}{ll}
\hline Parameters & Biological meanings \\
\hline$r$ & The prey intrinsic growth rate \\
$K$ & The prey carrying capacity \\
$a_{1}\left(\right.$ resp., $\left.a_{2}\right)$ & The maximum growth rate of predator (resp., top predator) \\
$e_{1}$ & The yield conversion factor for predator feeding on the prey \\
$e_{2}$ & The yield conversion factor for top predator feeding on the predator \\
$b_{1}\left(\right.$ resp., $\left.b_{2}\right)$ & The half saturation constant for predator (resp., top predator) \\
$\tilde{m}_{1}\left(\right.$ resp., $\left.\tilde{m}_{2}\right)$ & The death rate of predator (resp., top predator) \\
$d_{1}\left(\right.$ resp., $\left.d_{2}, d_{3}\right)$ & The diffusivity of the prey (resp., predator, top predator) \\
\hline
\end{tabular}

distinct feature of the simple food chain is secondary extinction because of trophic cascading effect [13, 14]. That means if one species dies out, all the species at higher trophic levels die out as well. Especially, there has been considerable interest in ratio-dependent food chain model for last two decades $[9,10,17,18,20,29]$. Peng et al. [18] investigated the following coupled reaction diffusion food chain model with ratio-dependent functional response:

$$
\begin{cases}\widetilde{N}_{t}-d_{1} \Delta \widetilde{N}=r \widetilde{N}\left(1-\frac{\widetilde{N}}{K}\right)-\frac{1}{e_{1}} \frac{a_{1} \widetilde{N} \widetilde{P}}{\widetilde{N}+b_{1} \widetilde{P}}, & t>0, x \in \Omega, \\ \widetilde{P}_{t}-d_{2} \Delta \widetilde{P}=\frac{a_{1} \widetilde{N} \widetilde{P}}{\widetilde{N}+b_{1} \widetilde{P}}-\tilde{m}_{1} \widetilde{P}-\frac{1}{e_{2}} \frac{a_{2} \widetilde{P} \widetilde{P}}{\widetilde{P}+b_{2} \widetilde{Z}}, & t>0, x \in \Omega, \\ \widetilde{Z}_{t}-d_{3} \Delta \widetilde{Z}=\frac{a_{2} \widetilde{P} \widetilde{Z}}{\widetilde{P}+b_{2} \widetilde{Z}}-\tilde{m}_{2} \widetilde{Z}, & t>0, x \in \Omega, \\ \frac{\partial \widetilde{N}(t, x)}{\partial \nu}=\frac{\partial \widetilde{P}(t, x)}{\partial \nu}=\frac{\partial \widetilde{Z}(t, x)}{\partial \nu}=0, & t \geq 0, x \in \partial \Omega, \\ \widetilde{N}(0, x)=\tilde{\phi}_{1}(x), \widetilde{P}(0, x)=\tilde{\phi}_{2}(x), \widetilde{Z}(0, x)=\tilde{\phi}_{3}(x), & x \in \Omega .\end{cases}
$$

Here, $\Omega$ is a fixed bounded open domain in $\mathbb{R}^{N}$ with smooth boundary at any given time, and $\nu$ is the outward unit normal vector on $\partial \Omega$. For $i=1,2,3$, the initial data are continuous functions and satisfy $\tilde{\phi}_{i}(x) \geq 0$, $\not \equiv 0$ for $x \in \Omega$. Parameters values are assigned according to biological meanings in Table 1 .

Taking into account the spatial spreading, we suppose that the prey and predator are native and the top predator is invasive. At initial time $(t=0)$, the prey and predator live in the whole line $\mathbb{R}$, the top predator exists in a bounded interval and spreads from double boundaries to expand its habitats. That is, it will move outward along the unknown curves (free boundaries) as time $t$ increases. To understand the dynamics of these three species and free boundaries, we formulate the following free boundary problem

$$
\begin{cases}\widetilde{N}_{t}-d_{1} \widetilde{N}_{x x}=\widetilde{N}\left(r-\frac{r}{K} \widetilde{N}-\frac{1}{e_{1}} \frac{a_{1} \widetilde{P}}{\widetilde{N}+b_{1} \widetilde{P}}\right), & t>0, x \in \mathbb{R} \\ \widetilde{P}_{t}-d_{2} \widetilde{P}_{x x}=\widetilde{P}\left(\frac{a_{1} \widetilde{N}}{\widetilde{N}+b_{1} \widetilde{P}}-\tilde{m}_{1}-\frac{1}{e_{2}} \frac{a_{2} \widetilde{Z}+b_{2} \widetilde{Z}}{\widetilde{P}}\right), & t>0, x \in \mathbb{R} \\ \widetilde{Z}_{t}-d_{3} \widetilde{Z}_{x x}=\widetilde{Z}\left(\frac{a_{2}}{\widetilde{P}+b_{2} \widetilde{Z}}-\tilde{m}_{2}\right), & t>0, \tilde{g}(t)<x<\tilde{h}(t) \\ Z(t, x)=0, & t \geq 0, x \notin(\tilde{g}(t), \tilde{h}(t)) \\ \tilde{g}^{\prime}(t)=-\tilde{\mu} \widetilde{Z}_{x}(t, \tilde{g}(t)), \tilde{h}^{\prime}(t)=-\tilde{\mu} \widetilde{Z}_{x}(t, \tilde{h}(t)), & t \geq 0, \\ \widetilde{N}(0, x)=\tilde{\phi}_{1}(x), \widetilde{P}(0, x)=\tilde{\phi}_{2}(x), & x \in \mathbb{R}, \\ \tilde{g}(0)=-\tilde{h}_{0}, \tilde{h}(0)=\tilde{h}_{0}, \tilde{Z}(0, x)=\tilde{\phi}_{3}(x), & -\tilde{h}_{0} \leq x \leq \tilde{h}_{0}\end{cases}
$$

where $\tilde{g}(t)$ and $\tilde{h}(t)$ represent the left and right moving boundaries, respectively; $r, K, d_{i}, a_{i}, b_{i}, e_{i}, \tilde{m}_{i}$, $\tilde{\mu}$, and $\tilde{h}_{0}$ are given positive constants for $i=1,2$. The free boundary conditions $\tilde{g}^{\prime}(t)=-\tilde{\mu} \widetilde{Z}_{x}(t, \tilde{g}(t))$ and 
$\tilde{h}^{\prime}(t)=-\tilde{\mu} \widetilde{Z}_{x}(t, \tilde{h}(t))$ are special cases of the well-known Stefan type conditions, and their ecological background and derivation can be referred to $[2,15,28]$.

It is worthwhile to mention free boundary problems have been introduced to describe the population spreading process. This paper can be seen as further research of [33], in which the authors studied a ratio-dependent preypredator model with a free boundary. More precisely, Du and Lin [6] firstly discussed a free boundary problem for the diffusive one-species Logistic model. They investigated the existence and uniqueness, regularity and uniform estimates, and long-time behavior of global solution. Some sufficient conditions for spreading and vanishing were also established. When spreading occurs, they provided some estimates of asymptotic spreading speeds of the free boundary. For additional relevant works in this direction can be found in $[2,3,5,7,12,15,21-23]$. Free boundary problems for the diffusive two-species competition models and prey-predator models have been systematically investigated in literatures, refer to $[8,24,25,27,28,30,33,34]$. Recently, a free boundary problem for the diffusive three-species model has been studied in [31].

For simplicity, we non-dimensionalize problem (1.3) with the following scaling:

$$
N=\frac{1}{K} \widetilde{N}, \quad P=\frac{b_{1}}{K} \widetilde{P}, \quad Z=\frac{b_{1} b_{2}}{K} \widetilde{Z}, \quad \bar{t}=t r, \quad \bar{x}=\sqrt{\frac{r}{d_{3}}} x
$$

Dropping the caps of $t$ and $x$, then problem (1.3) is rewritten in the following form:

$$
\begin{cases}N_{t}-D_{1} N_{x x}=N\left(1-N-c_{1} \frac{P}{N+P}\right), & t>0, x \in \mathbb{R}, \\ P_{t}-D_{2} P_{x x}=P\left(k_{1} \frac{N}{N+P}-m_{1}-c_{2} \frac{Z}{P+Z}\right), & t>0, x \in \mathbb{R}, \\ Z_{t}-Z_{x x}=Z\left(k_{2} \frac{P}{P+Z}-m_{2}\right), & t>0, g(t)<x<h(t), \\ Z(t, x)=0, & t \geq 0, x \notin(g(t), h(t)), \\ g^{\prime}(t)=-\mu Z_{x}(t, g(t)), h^{\prime}(t)=-\mu Z_{x}(t, h(t)), & t \geq 0, \\ N(0, x)=\phi_{1}(x), P(0, x)=\phi_{2}(x), & x \in \mathbb{R}, \\ g(0)=-h_{0}, h(0)=h_{0}, Z(0, x)=\phi_{3}(x), & -h_{0} \leq x \leq h_{0},\end{cases}
$$

where

$$
\begin{aligned}
& D_{1}=\frac{d_{1}}{d_{3}}, D_{2}=\frac{d_{2}}{d_{3}}, c_{1}=\frac{a_{1}}{r e_{1} b_{1}}, c_{2}=\frac{a_{2}}{r e_{2} b_{2}}, k_{1}=\frac{a_{1}}{r}, \\
& k_{2}=\frac{a_{2}}{r}, m_{1}=\frac{\tilde{m}_{1}}{r}, m_{2}=\frac{\tilde{m}_{2}}{r}, \mu=\frac{\tilde{\mu} K}{b_{1} b_{2} \sqrt{r d_{3}}}, h_{0}=\sqrt{\frac{r}{d_{3}}} \tilde{h}_{0}, \\
& \phi_{1}(x)=\frac{1}{K} \tilde{\phi}_{1}\left(\sqrt{\frac{d_{3}}{r}} x\right), \phi_{2}(x)=\frac{b_{1}}{K} \tilde{\phi}_{2}\left(\sqrt{\frac{d_{3}}{r}} x\right), \phi_{3}(x)=\frac{b_{1} b_{2}}{K} \tilde{\phi}_{3}\left(\sqrt{\frac{d_{3}}{r}} x\right) .
\end{aligned}
$$

Furthermore, we suppose that $\phi_{1}(x), \phi_{2}(x)$, and $\phi_{3}(x)$ satisfy

$$
\left\{\begin{array}{l}
\phi_{1}(x), \phi_{2}(x) \in C_{b}(\mathbb{R}), \phi_{1}(x), \phi_{2}(x)>0 \text { in } \mathbb{R}, \\
\phi_{3}(x) \in W_{p}^{2}\left(\left[-h_{0}, h_{0}\right]\right), \phi_{3}\left( \pm h_{0}\right)=0, \phi_{3}(x)>0 \text { in }\left(-h_{0}, h_{0}\right),
\end{array}\right.
$$

where $p>3, C_{b}(\mathbb{R})$ is the space of continuous and bounded functions in $\mathbb{R}$.

Inspired by $[10,29]$, we may define that $E_{0}=(0,0,0)$ is an trivial steady state of (1.4). Besides, problem (1.4) also admits the semi-trivial steady states $E_{1}=(1,0,0)$ and

$$
E_{2}=\left(\frac{k_{1}-c_{1}\left(k_{1}-m_{1}\right)}{k_{1}}, \frac{\left(k_{1}-m_{1}\right)\left[k_{1}-c_{1}\left(k_{1}-m_{1}\right)\right]}{k_{1} m_{1}}, 0\right) \stackrel{\text { def }}{=}\left(N_{2}^{*}, P_{2}^{*}, 0\right)
$$


if and only if $0<c_{1}\left(k_{1}-m_{1}\right)<k_{1}$. It is easily shown that (1.4) has a unique positive constant steady state $E_{3} \stackrel{\text { def }}{=}\left(N_{3}^{*}, P_{3}^{*}, Z_{3}^{*}\right)$ if and only if the following are satisfied:

$$
\left(\boldsymbol{H}_{1}\right) k_{2}>m_{2}, \mathcal{A}>1 \text { and } 0<c_{1}<\frac{\mathcal{A}}{\mathcal{A}-1},
$$

where

$$
\mathcal{A}=\frac{k_{1}}{c_{2} \frac{k_{2}-m_{2}}{k_{2}}+m_{1}} .
$$

Moreover, the unique positive constant steady state can be expressed as

$$
N_{3}^{*}=\frac{c_{1}+\mathcal{A}\left(1-c_{1}\right)}{\mathcal{A}}, \quad P_{3}^{*}=(\mathcal{A}-1) N_{3}^{*}, \quad Z_{3}^{*}=\frac{\left(k_{2}-m_{2}\right) P_{3}^{*}}{m_{2}} .
$$

In addition, we note that $k_{2}>m_{2}$ and $\mathcal{A}>1$ imply $k_{1}>m_{1}$.

In the absence of diffusion, Hsu et al. [10] dealt with problem (1.2). Obviously, the steady states $E_{0}$ and $E_{1}$ always exist. For some suitable conditions, they concluded that $E_{0}$ is globally asymptotically stable when $c_{1}>1$ and $E_{1}$ is globally asymptotically stable when $c_{1}<1$, please refer to Theorems 2.1 and 2.3 in [10]. Then, Peng et al. [18] investigated the stationary pattern of problem (1.2). They obtained that $E_{1}$ is globally asymptotically stable if $c_{1} \leq 1$ and $k_{1} \leq m_{1}$, see Proposition 3.1 in [18]. As shown in Proposition 3.1 in [18], if $k_{1} \leq m_{1}$ or $k_{2} \leq m_{2}$, the two predators or the top predator will face extinction, respectively. In this work, we mainly analyze that the influence of free boundaries on the spreading process of the top predator. Therefore, we only consider the assumptions that $k_{1}>m_{1}, k_{2}>m_{2}$, and $c_{1}<1$.

The organization of this paper is organized as follows. In Section 2, we first give the local existence and uniqueness of the solution to (1.4) and then show that the solution exists for all time $t \in(0, \infty)$. Section 3 is devoted to the long time behavior of $(N, P, Z)$. In Section 4, we shall provide the criteria governing spreading and vanishing. Furthermore, two numerical examples to illustrate the theoretical analysis are carried out in Section 5. The last section is a brief conclusion and discussion.

\section{Existence And uniqueness of the SOlution to PROBlem (1.4)}

In this section, we prove the following local existence and uniqueness result by applying the contraction mapping theorem and the upper and lower solutions method, and then we show the global existence via some suitable estimates. Define $g^{*}=-\mu \phi_{3}^{\prime}\left(-h_{0}\right), h^{*}=-\mu \phi_{3}^{\prime}\left(h_{0}\right)$ and

$$
\Sigma=\left\{D_{1}, D_{2}, k_{1}, k_{2}, c_{1}, c_{2}, m_{1}, m_{2}, \mu, h_{0}, g^{*}, h^{*},\left\|\phi_{1}(x)\right\|_{\infty},\left\|\phi_{2}(x)\right\|_{\infty},\left\|\phi_{3}(x)\right\|_{W_{p}^{2}\left(\left[-h_{0}, h_{0}\right]\right)}\right\} .
$$

Theorem 2.1. For any given $\phi_{1}(x), \phi_{2}(x), \phi_{3}(x)$ satisfying $(1.5), \alpha \in(0,1)$ and $p>\frac{3}{1-\alpha}$, there is a $T>0$ such that problem (1.4) admits a unique solution

$$
(N, P, Z, g, h) \in\left[C_{b}\left(\bar{I}_{T}^{\infty}\right) \cap C_{l o c}^{1+\frac{\alpha}{2}, 2+\alpha}\left(I_{T}^{\infty}\right)\right]^{2} \times\left(W_{p}^{1,2}\left(I_{T}^{(g, h)}\right) \cap C^{\frac{1+\alpha}{2}, 1+\alpha}\left(\bar{I}_{T}^{(g, h)}\right)\right) \times\left[C^{1+\frac{\alpha}{2}}([0, T])\right]^{2},
$$

where $I_{T}^{\infty}=\left\{(t, x) \in \mathbb{R}^{2}: t \in(0, T], x \in \mathbb{R}\right\}, I_{T}^{(g, h)}=\left\{(t, x) \in \mathbb{R}^{2}: t \in(0, T], x \in(g(t), h(t))\right\}$. Moreover,

$$
\|Z\|_{W_{p}^{1,2}\left(I_{T}^{(g, h)}\right)}+\|Z\|_{C^{\frac{1+\alpha}{2}, 1+\alpha}\left(\bar{I}_{T}^{(g, h)}\right)}+\|g\|_{C^{1+\frac{\alpha}{2}([0, T])}}+\|h\|_{C^{1+\frac{\alpha}{2}([0, T])}} \leq C,
$$

here positive constants $C$ and $T$ only depend on $\Sigma, \alpha$, and $p$. 
Proof. The proof is motivated by $[23,28,32]$. Firstly, we straighten the free boundaries. Let

$$
y=\frac{2 x-g(t)-h(t)}{h(t)-g(t)}
$$

so that the transformation $(t, x) \rightarrow(t, y)$ helps us straighten the left free boundary $x=g(t)$ to the line $y=-1$ and the right free boundary $x=h(t)$ to the line $y=1$. Set

$$
\begin{aligned}
Q(t, y) & =N\left(t, \frac{1}{2}[(h(t)-g(t)) y+h(t)+g(t)]\right), \\
R(t, y) & =P\left(t, \frac{1}{2}[(h(t)-g(t)) y+h(t)+g(t)]\right), \\
W(t, y) & =Z\left(t, \frac{1}{2}[(h(t)-g(t)) y+h(t)+g(t)]\right) .
\end{aligned}
$$

Therefore, the free boundary problem (1.4) is equivalent to

$$
\begin{gathered}
\begin{cases}Q_{t}-D_{1} A^{2}(t) Q_{y y}-B(t, y) Q_{y}=Q\left(1-Q-c_{1} \frac{R}{Q+R}\right), & t>0, y \in \mathbb{R}, \\
R_{t}-D_{2} A^{2}(t) R_{y y}-B(t, y) R_{y}=R\left(k_{1} \frac{Q}{Q+R}-m_{1}-c_{2} \frac{W}{R+W}\right), & t>0, y \in \mathbb{R}, \\
Q(0, y)=\phi_{1}\left(h_{0} y\right), R(0, y)=\phi_{2}\left(h_{0} y\right), & y \in \mathbb{R},\end{cases} \\
\begin{cases}W_{t}-A^{2}(t) W_{y y}-B(t, y) W_{y}=W\left(k_{2} \frac{R}{R+W}-m_{2}\right), & t>0,|y|<1, \\
W(t, y)=0, & t \geq 0,|y| \geq 1, \\
W(0, y)=\phi_{3}\left(h_{0} y\right), & |y| \leq 1,\end{cases} \\
\begin{cases}g(0)=-h_{0}, \quad g^{\prime}(t)=-\mu A(t) W_{y}(t,-1), & t \geq 0, \\
h(0)=h_{0}, \quad h^{\prime}(t)=-\mu A(t) W_{y}(t, 1), & t \geq 0,\end{cases}
\end{gathered}
$$

where

$$
A(t)=\frac{2}{h(t)-g(t)}, \quad B(t, y)=\frac{h^{\prime}(t)+g^{\prime}(t)}{h(t)-g(t)}+\frac{h^{\prime}(t)-g^{\prime}(t)}{h(t)-g(t)} y
$$

The rest of the proof is similar to that of Theorem 1.1 in [23] and Theorem 2.1 in [28]. By using the upper and lower solutions method, we may get the local existence of the solution $(Q, R, W)$ to the initial-boundary value problems (2.2) and (2.3) with fixed boundary for given $g(t)$ and $h(t)$. Then, the local existence and uniqueness of the solution $(Q, R, W, g, h)$ to $(2.2),(2.3)$, and (2.4) is obtained by the contraction mapping theorem, $L^{p}$ theory, Schauder theory and the Sobolev imbedding theorem [11]. See also Du and Lin [6], Wang and Zhang [24]. We omit the details.

To show the global existence of the solution, we need the following priori estimates.

Lemma 2.2. Assume that $k_{1}>m_{1}$ and $k_{2}>m_{2}$. Let $T>0$ and $(N, P, Z, g, h)$ be a solution of (1.4) defined for $t \in(0, T)$. Then there exist constants $M_{1}, M_{2}, M_{3}$ and $M_{4}$ independent of $T$ such that

$$
0<N \leq \max \left\{\left\|\phi_{1}\right\|_{\infty}, 1\right\} \stackrel{\text { def }}{=} M_{1} \quad \text { in }(0, T) \times \mathbb{R}
$$




$$
\begin{aligned}
& 0<P \leq \max \left\{\left\|\phi_{2}\right\|_{\infty},\left(\frac{k_{1}}{m_{1}}-1\right) M_{1}\right\} \stackrel{\text { def }}{=} M_{2} \quad \text { in }(0, T) \times \mathbb{R}, \\
& 0<Z \leq \max \left\{\left\|\phi_{3}\right\|_{\infty}, \quad\left(\frac{k_{2}}{m_{2}}-1\right) M_{2}\right\} \stackrel{\text { def }}{=} M_{3} \quad \text { in }(0, T) \times(g(t), h(t)), \\
& -2 \mu M_{3} M_{4} \leq g^{\prime}(t)<0, \quad 0<h^{\prime}(t) \leq 2 \mu M_{3} M_{4} \quad \text { in }(0, T),
\end{aligned}
$$

where $M_{4}=\max \left\{\frac{1}{h_{0}}, \sqrt{\frac{k_{2}-m_{2}}{2}}, \frac{4\left\|\phi_{3}\right\|_{C^{1}\left(\left[-h_{0}, h_{0}\right]\right)}}{3 M_{3}}\right\}$.

Proof. Firstly, as long as the solution exists, one can see that $N, P>0$ in $(0, T) \times \mathbb{R}$ and $Z>0$ in $(0, T) \times$ $(g(t), h(t))$ by the strong maximum principle. Note that $N, P$ and $Z$ satisfy

$$
\begin{cases}N_{t}-D_{1} N_{x x} \leq N(1-N), & 0<t<T, x \in \mathbb{R}, \\ P_{t}-D_{2} P_{x x} \leq P\left(k_{1} \frac{N}{N+P}-m_{1}\right), & 0<t<T, x \in \mathbb{R}, \\ Z_{t}-Z_{x x}=Z\left(k_{2} \frac{P}{P+Z}-m_{2}\right), & 0<t<T, g(t)<x<h(t), \\ Z(t, x)=0, & 0 \leq t<T, x \notin(g(t), h(t)), \\ g^{\prime}(t)=-\mu Z_{x}(t, g(t)), \quad h^{\prime}(t)=-\mu Z_{x}(t, h(t)), & 0 \leq t<T, \\ N(0, x)=\phi_{1}(x), \quad P(0, x)=\phi_{2}(x), & x \in \mathbb{R}, \\ Z(0, x)=\phi_{3}(x), & -h_{0} \leq x \leq h_{0} .\end{cases}
$$

In view of the comparison principle, $N \leq M_{1}, P \leq M_{2}$ and $Z \leq M_{3}$.

To prove $h^{\prime}(t)>0$ for $0<t<T$, set

$$
y=\frac{x}{h(t)}, \quad Q(t, y)=N(t, x), \quad R(t, y)=P(t, x), \quad W(t, y)=Z(t, x),
$$

and yet straighten the free boundary $x=h(t)$ to the line $y=1$. Hence, $Q, R$ and $W$ obey

$$
\begin{cases}W_{t}-\psi(t) W_{y y}-\varphi(t, y) W_{y}=W\left(k_{2} \frac{R}{R+W}-m_{2}\right), & 0<t<T, 0<y<1 \\ W(t, 0)>0, \quad W(t, y)=0, & 0 \leq t<T, y \geq 1 \\ W(0, y)=\phi_{3}\left(h_{0} y\right), & 0 \leq y \leq 1\end{cases}
$$

where $\psi(t)=\frac{1}{h^{2}(t)}, \varphi(t, y)=\frac{y h^{\prime}(t)}{h(t)}$. Then, $W>0$ in $(0, T) \times[0,1)$ and the Hopf boundary lemma imply $W_{y}(t, 1)<0$ for $0<t<T$. With the relation $Z_{x}=\frac{W_{y}}{h(t)}$, it follows that $Z_{x}(t, h(t))<0$. Consequently, $h^{\prime}(t)>0$ for $0<t<T$. Likewise, $g^{\prime}(t)<0$ for $0<t<T$.

We now claim $h^{\prime}(t) \leq 2 \mu M_{3} M_{4}$. To verify this assertion, compare $Z$ with the following auxiliary function. Define

$$
\Gamma=\left\{(t, x) \in \mathbb{R}^{2}: 0<t<T, h(t)-M_{4}^{-1}<x<h(t)\right\}
$$

and construct an auxiliary function

$$
V(t, x)=M_{3}\left[2 M_{4}(h(t)-x)-M_{4}^{2}(h(t)-x)^{2}\right] .
$$

Direct calculations give that, for $(t, x) \in \Gamma$,

$$
V_{t}=2 M_{3} M_{4} h^{\prime}(t)\left[1-M_{4}(h(t)-x)\right] \geq 0, \quad-V_{x x}=2 M_{3} M_{4}^{2},
$$




$$
M_{3}\left(k_{2}-m_{2}\right) \geq Z\left(k_{2} \frac{P}{P+Z}-m_{2}\right)
$$

Therefore, we observe that

$$
V_{t}-V_{x x} \geq 2 M_{3} M_{4}^{2} \geq M_{3}\left(k_{2}-m_{2}\right) \geq Z\left(k_{2} \frac{P}{P+Z}-m_{2}\right) \text { in } \Gamma
$$

provided $M_{4}^{2} \geq \frac{k_{2}-m_{2}}{2}$. Additionally, for $0<t<T$,

$$
V\left(t, h(t)-M_{4}^{-1}\right)=M_{3} \geq Z\left(t, h(t)-M_{4}^{-1}\right), \quad V(t, h(t))=0=Z(t, h(t)) .
$$

Consequently, we expect to find some $M_{4}$ independent of $T$ such that

$$
V(0, x) \geq \phi_{3}(x) \text { for } x \in\left[h_{0}-M_{4}^{-1}, h_{0}\right] \text {. }
$$

Thus, from (2.5), (2.6), (2.7), and the comparison principle, we may deduce $V \geq Z$ in $\Gamma$. Then,

$$
h^{\prime}(t)=-\mu Z_{x}(t, h(t)) \leq-\mu V_{x}(t, h(t))=2 \mu M_{3} M_{4}, \quad 0<t<T .
$$

It remains to show that (2.7) holds. To this aim, choose

$$
M_{4}=\max \left\{\frac{1}{h_{0}}, \sqrt{\frac{k_{2}-m_{2}}{2}}, \frac{4\left\|\phi_{3}\right\|_{C^{1}\left(\left[-h_{0}, h_{0}\right]\right)}}{3 M_{3}}\right\},
$$

and divide $\left[h_{0}-M_{4}^{-1}, h_{0}\right]$ into two subsets: $\left[h_{0}-M_{4}^{-1}, h_{0}-\left(2 M_{4}\right)^{-1}\right]$ and $\left[h_{0}-\left(2 M_{4}\right)^{-1}, h_{0}\right]$. For $x \in$ $\left[h_{0}-\left(2 M_{4}\right)^{-1}, h_{0}\right]$, we have

$$
V_{x}(0, x)=-2 M_{3} M_{4}\left[1-M_{4}\left(h_{0}-x\right)\right] \leq-M_{3} M_{4} \leq \phi_{3}^{\prime}(x) .
$$

Combining this with $V\left(0, h_{0}\right)=\phi_{3}\left(h_{0}\right)=0$, derives

$$
V(0, x) \geq \phi_{3}(x) \text { for } x \in\left[h_{0}-\left(2 M_{4}\right)^{-1}, h_{0}\right]
$$

As $M_{4} \geq \frac{4\left\|\phi_{3}\right\|_{C^{1}\left(\left[-h_{0}, h_{0}\right]\right)}}{3 M_{3}}$, for $x \in\left[h_{0}-M_{4}^{-1}, h_{0}-\left(2 M_{4}\right)^{-1}\right]$, we have

$$
V(0, x) \geq \frac{3}{4} M_{3} \geq\left\|\phi_{3}(x)\right\|_{C^{1}\left(\left[-h_{0}, h_{0}\right]\right)} M_{4}^{-1} \geq \phi_{3}(x) .
$$

So, (2.7) holds. It means that (2.8) is valid. Similarly, $g^{\prime}(t) \geq-2 \mu M_{3} M_{4}$. The proof is finished.

Theorem 2.3. Assume that $k_{1}>m_{1}$ and $k_{2}>m_{2}$. Then the solution $(N, P, Z, g, h)$ of (1.4) exists and is unique for all $t \in(0, \infty)$.

Proof. Let $T_{\max }$ be the maximum existence time of $(N, P, Z, g, h)$. Taking advantage of Theorem 2.1, we observe $T_{\max }>0$. We now wish to show $T_{\max }=\infty$.

Suppose that $T_{\max }<\infty$. In terms of Lemma 2.2, we have

$$
0 \leq N \leq M_{1}, 0 \leq P \leq M_{2} \quad \text { in }\left[0, T_{\max }\right) \times \mathbb{R}
$$




$$
\begin{aligned}
& 0 \leq Z \leq M_{3} \quad \text { in }\left[0, T_{\max }\right) \times[g(t), h(t)] \\
& 0 \leq h^{\prime}(t) \leq 2 \mu M_{3} M_{4}, h_{0} \leq h(t) \leq h_{0}+2 \mu M_{3} M_{4} t \quad \text { in }\left[0, T_{\max }\right) \\
& -2 \mu M_{3} M_{4} \leq g^{\prime}(t) \leq 0,-2 \mu M_{3} M_{4} t-h_{0} \leq g(t) \leq-h_{0} \quad \text { in }\left[0, T_{\max }\right)
\end{aligned}
$$

Choose $\sigma \in\left(0, T_{\max }\right)$. Arguing as the same as the proof of Theorem 2.1, one can see that $N(t, \cdot), P(t, \cdot) \in$ $C_{\text {loc }}^{1+\frac{\alpha}{2}}(\mathbb{R}),\|N(t, \cdot)\|_{L^{\infty}}(\mathbb{R}) \leq M_{1}$ and $\|P(t, \cdot)\|_{L^{\infty}}(\mathbb{R}) \leq M_{2}$ for $t \in\left[\sigma, T_{\max }\right)$. In addition, there is a positive constant $C$ independent of $T_{\max }$ such that $\|Z(t, \cdot)\|_{W_{p}^{2}([g(t), h(t)])} \leq C$ for $t \in\left[\sigma, T_{\max }\right)$. Then, following the proof of Theorem 2.1 again, there exists $\tau>0$ independent of $T_{\max }$ such that the solution of (1.4) with initial time $T_{\max }-\frac{\tau}{2}$ can be extended uniquely to the time $T_{\max }-\frac{\tau}{2}+\tau$. This means that the solution of (1.4) can be extended as long as $N, P$ and $Z$ remain bounded, which is in contradiction with our hypothesis. Thus, $T_{\max }=\infty$. The proof is complete.

\section{LONG TIME BEHAVior of $(N, P, Z)$}

Thanks to Lemma 2.2, there exist $g_{\infty} \in[-\infty, 0)$ and $h_{\infty} \in(0, \infty]$ such that $\lim _{t \rightarrow \infty} g(t)=g_{\infty}$ and $\lim _{t \rightarrow \infty} h(t)=h_{\infty}$. Then, the subsequent analysis is divided into two parts: vanishing case $\left(h_{\infty}-g_{\infty}<\infty\right)$ and spreading case $\left(h_{\infty}-g_{\infty}=\infty\right)$.

We now present the following results that will be used frequently.

Proposition 3.1. ([28], Prop. 8.1) Let $d, \beta$ and $\theta$ be fixed positive constants, and $\kappa$ be a non-negative constant. For any given $\epsilon, L>0$, there exist $T_{\epsilon}>0$ and $l_{\epsilon}>\max \left\{L, \frac{\pi}{2} \sqrt{\frac{d}{\beta}}\right\}$ such that when the continuous and nonnegative function $\omega(t, x)$ satisfies

$$
\begin{cases}\omega_{t}-d \omega_{x x} \geq(\leq) \omega(\beta-\theta \omega), & t>0,-l_{\epsilon}<x<l_{\epsilon} \\ \omega(0, x)>0, & -l_{\epsilon}<x<l_{\epsilon}\end{cases}
$$

and for $t>0, \omega\left(t, \pm l_{\epsilon}\right) \geq(\leq) \kappa$ if $\kappa>0$, while $\omega\left(t, \pm l_{\epsilon}\right) \geq(=) 0$ if $\kappa=0$, then we have

$$
\omega(t, x)>\frac{\beta}{\theta}-\epsilon \quad\left(\omega(t, x)<\frac{\beta}{\theta}+\epsilon\right), \quad \forall t \geq T_{\epsilon}, x \in[-L, L] .
$$

This implies

$$
\liminf _{t \rightarrow \infty} \omega(t, x)>\frac{\beta}{\theta}-\epsilon \quad\left(\limsup _{t \rightarrow \infty} \omega(t, x)<\frac{\beta}{\theta}+\epsilon\right) \text { uniformly on }[-L, L] .
$$

Lemma 3.2. Assume that $\beta>\theta$. Let $d, \beta$, $\varrho$ and $\theta$ be fixed positive constants, and $\kappa$ be a non-negative constant. For any given $\epsilon>0$ and $L>0$, there exist $T_{\epsilon}>0$ and $l_{\epsilon}>\max \left\{L, \frac{\pi}{2} \sqrt{\frac{d}{\beta-\theta}}\right\}$ such that when the continuous and non-negative function $\omega(t, x)$ satisfies

$$
\begin{cases}\omega_{t}-d \omega_{x x} \geq(\leq) \omega\left(\frac{\beta \varrho}{\varrho+\omega}-\theta\right), & t>0,-l_{\epsilon}<x<l_{\epsilon} \\ \omega(0, x)>0, & -l_{\epsilon}<x<l_{\epsilon}\end{cases}
$$

and for $t>0, \omega\left(t, \pm l_{\epsilon}\right) \geq(\leq) \kappa$ if $\kappa>0$, while $\omega\left(t, \pm l_{\epsilon}\right) \geq(=) 0$ if $\kappa=0$. Then, we obtain

$$
\omega(t, x)>\frac{\beta \varrho}{\theta}-\varrho-\epsilon \quad\left(\omega(t, x)<\frac{\beta \varrho}{\theta}-\varrho+\epsilon\right), \quad \forall t \geq T_{\epsilon}, x \in[-L, L],
$$


which implies

$$
\liminf _{t \rightarrow \infty} \omega(t, x)>\frac{\beta \varrho}{\theta}-\varrho-\epsilon \quad\left(\limsup _{t \rightarrow \infty} \omega(t, x)<\frac{\beta \varrho}{\theta}-\varrho+\epsilon\right) \text { uniformly on }[-L, L] .
$$

The proof of Lemma 3.2 is similar to that in Lemmas A.5 and A.4 in [33] with some modifications and we omit it here.

\subsection{Vanishing case: $h_{\infty}-g_{\infty}<\infty$}

To discuss the vanishing case, we first exhibit the following estimate and remember a basic result.

Theorem 3.3. Let $(N, P, Z, g, h)$ be the unique global solution of (1.4). If $h_{\infty}-g_{\infty}<\infty$, then there is a constant $\xi>0$ such that

$$
\begin{aligned}
& \|Z(t, \cdot)\|_{C^{1}([g(t), h(t)])} \leq \xi, \quad \forall t>1, \\
& \lim _{t \rightarrow \infty} g^{\prime}(t)=\lim _{t \rightarrow \infty} h^{\prime}(t)=0 .
\end{aligned}
$$

Proof. By similar arguments in the proof of Theorem 2.1, we adopt the following transformation

$$
y=\frac{2 x-g(t)-h(t)}{h(t)-g(t)}, \quad Q(t, y)=N(t, x), \quad R(t, y)=P(t, x), \quad W(t, y)=Z(t, x)
$$

to straighten the free boundaries $x=h(t)$ and $x=g(t)$ to the lines $y=1$ and $y=-1$. The direct calculations show that $R, W$ obey (2.3) and $g, h$ satisfy (2.4). Consequently, in light of Proposition 7.1 from [28], there exists a constant $\xi_{0}>0$ such that

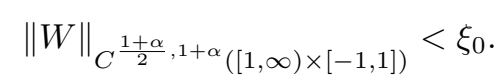

With $h_{\infty}-g_{\infty}<\infty$ and the relation $Z_{x}(t, x)=\frac{2}{h(t)-g(t)} W_{y}(t, y)$, we derive (3.1).

We now check $\lim _{t \rightarrow \infty} g^{\prime}(t)=0$. Recalling $(3.3),\left\|W_{y}(\cdot,-1)\right\|_{C^{\frac{\alpha}{2}}([1, \infty))}<\xi_{0}$ holds. Then, we may find a constant $M>0$ such that $-M \leq g^{\prime}(t)<0$ as in Lemma 2.2 and yet (2.4) yields

$$
g^{\prime}(t)=-\mu Z_{x}(t, g(t))=-\frac{2 \mu}{h(t)-g(t)} W_{y}(t,-1) .
$$

Combining all above, there exists a constant $\xi_{1}>0$ such that

$$
\left\|g^{\prime}\right\|_{C^{\frac{\alpha}{2}([1, \infty))}} \leq \xi_{1} .
$$

We have $g_{\infty}>-\infty$ and $h_{\infty}<\infty$ according to the condition $h_{\infty}-g_{\infty}<\infty$. Assume that $\lim _{t \rightarrow \infty} g^{\prime}(t)=0$ does not hold. Then, for some $\delta>0$, there exists a sequence $\left\{t_{n}\right\}$ such that $t_{n} \rightarrow \infty$ and $g^{\prime}\left(t_{n}\right) \rightarrow-\delta$ as $n \rightarrow \infty$. In terms of (3.4), we choose $\epsilon>0$ so small that $g^{\prime}(t) \leq-\frac{\delta}{2}$ for all $t \in\left[t_{n}-\epsilon, t_{n}+\epsilon\right] \subseteq[1, \infty)$ for all $n=1,2, \cdots$. Thus,

$$
\int_{1}^{\infty} g^{\prime}(\tau) \mathrm{d} \tau \leq \sum_{n=1}^{\infty} \int_{t_{n}-\epsilon}^{t_{n}+\epsilon}-\frac{\delta}{2} \mathrm{~d} t=-\infty .
$$


In addition,

$$
\int_{1}^{\infty} g^{\prime}(\tau) \mathrm{d} \tau=\lim _{t \rightarrow \infty} \int_{1}^{t} g^{\prime}(\tau) \mathrm{d} \tau=\lim _{t \rightarrow \infty} g(t)-g(1)=g_{\infty}-g(1)>-\infty
$$

This result contradicts with (3.5). Hence, $\lim _{t \rightarrow \infty} g^{\prime}(t)=0$ holds. Similarly, $\lim _{t \rightarrow \infty} h^{\prime}(t)=0$ can be proved. The proof is finished.

Lemma 3.4. ([26], Lem. 4.1) Let $d, C, \mu$ and $\rho_{0}$ be positive constants, $\omega \in W_{p}^{1,2}((0, T) \times(0, \rho(t)))$ for some $p>1$ and any $T>0$, and $\omega_{x} \in C([0, \infty) \times[0, \rho(t)]), \rho(t) \in C^{1}([0, \infty))$. If $(\omega, \rho)$ satisfies

$$
\begin{cases}\omega_{t}-d \omega_{x x} \geq-C \omega, & t>0,0<x<\rho(t) \\ \omega \geq 0, & t>0, x=0 \\ \omega=0, \rho^{\prime}(t) \geq-\mu \omega_{x}, & t>0, x=\rho(t) \\ \omega(0, x)=\omega_{0}(x) \geq, \not \equiv 0, & x \in\left(0, \rho_{0}\right) \\ \rho(0)=\rho_{0}, & \end{cases}
$$

and

$$
\begin{gathered}
\lim _{t \rightarrow \infty} \rho(t)=\rho_{\infty}<\infty, \quad \lim _{t \rightarrow \infty} \rho^{\prime}(t)=0, \\
\|\omega(t, \cdot)\|_{C^{1}[0, \rho(t)]} \leq \xi, \quad \forall t>1
\end{gathered}
$$

for some constant $\xi>0$. Then

$$
\lim _{t \rightarrow \infty} \max _{0 \leq x \leq \rho(t)} \omega(t, x)=0
$$

Theorem 3.5. Assume that $k_{1}>m_{1}$ and $c_{1}<1$. Let $(N, P, Z, g, h)$ be the unique global solution of (1.4). If $h_{\infty}-g_{\infty}<\infty$, then

$$
\begin{aligned}
& \lim _{t \rightarrow \infty} N(t, x)=N_{2}^{*} \text { uniformly in any compact subset of } \mathbb{R}, \\
& \lim _{t \rightarrow \infty} P(t, x)=P_{2}^{*} \text { uniformly in any compact subset of } \mathbb{R}, \\
& \lim _{t \rightarrow \infty}\|Z(t, x)\|_{C([g(t), h(t)])}=0 .
\end{aligned}
$$

Proof. First, from Theorem 3.3 and Lemma 3.4, we deduce (3.8). Fix $0<\eta \ll 1$. In view of $Z(t, x)=0$ for $t>0$ and $x \notin(g(t), h(t))$, there exists $T_{0}>0$ such that

$$
Z(t, x)<\eta \text { in }\left[T_{0}, \infty\right) \times \mathbb{R}
$$

Next, we prove (3.6) and (3.7) by constructing the following iteration sequences $\left\{\bar{N}_{i}\right\},\left\{\bar{P}_{i}\right\},\left\{\underline{N}_{i}\right\}$ and $\left\{\underline{P}_{i}\right\}$.

Step 1: The constructions of $\bar{N}_{1}$ and $\bar{P}_{1}$. 
Take $M=\max \left\{M_{1}, M_{2}, M_{3}\right\}$, where $M_{i}(i=1,2,3)$ is determined by Lemma 2.2. It is easy to see that $N$ satisfies

$$
\begin{cases}N_{t}-D_{1} N_{x x} \leq N(1-N), & t>0, x \in \mathbb{R} \\ N(t, x) \leq M, & t \geq 0, x \in \mathbb{R} \\ N(0, x)=\phi_{1}(x)>0, & x \in \mathbb{R}\end{cases}
$$

Using the comparison principle, we observe

$$
\limsup _{t \rightarrow \infty} N(t, x) \leq 1 \stackrel{\text { def }}{=} \bar{N}_{1} \text { uniformly on the compact subset of } \mathbb{R} \text {. }
$$

Set $L \gg 1$ and $0<\epsilon, \delta \ll 1$. Let $l_{\epsilon}$ be given as in Lemma 3.2 with $\beta=k_{1}, \varrho=\bar{N}_{1}+\delta, \theta=m_{1}$ and $\kappa=M$. Owing to (3.10), there is $T_{1}>T_{0}$ such that

$$
N(t, x) \leq \bar{N}_{1}+\delta \text { in }\left[T_{1}, \infty\right) \times\left[-l_{\epsilon}, l_{\epsilon}\right] .
$$

Hence, $P$ obeys

$$
\begin{cases}P_{t}-D_{2} P_{x x} \leq P\left(k_{1} \frac{\bar{N}_{1}+\delta}{\bar{N}_{1}+\delta+P}-m_{1}\right), & t>T_{1},-l_{\epsilon}<x<l_{\epsilon} \\ P\left(t, \pm l_{\epsilon}\right) \leq M, & t \geq T_{1} \\ P\left(T_{1}, x\right)>0, & -l_{\epsilon} \leq x \leq l_{\epsilon}\end{cases}
$$

Applying Lemma 3.2, we discover

$$
\limsup _{t \rightarrow \infty} P(t, x)<\left(\frac{k_{1}}{m_{1}}-1\right)\left(\bar{N}_{1}+\delta\right)+\epsilon \text { uniformly on }[-L, L] .
$$

In consideration of the arbitrariness of $L, \epsilon$ and $\delta$, we obtain

$$
\limsup _{t \rightarrow \infty} P(t, x) \leq\left(\frac{k_{1}}{m_{1}}-1\right) \bar{N}_{1} \stackrel{\text { def }}{=} \bar{P}_{1} \text { uniformly on the compact subset of } \mathbb{R} \text {. }
$$

Step 2: The constructions of $\underline{N}_{1}$ and $\underline{P}_{1}$.

Let $L \gg 1,0<\epsilon, \delta \ll 1$, and $l_{\epsilon}$ be governed by Proposition 3.1 with $\beta=1-c_{1}-\delta, \theta=1$ and $\kappa=0$. Therefore, there exists $T_{2}>T_{1}$ such that $N$ satisfies

$$
\begin{cases}N_{t}-D_{1} N_{x x} \geq N\left(1-N-c_{1}-\delta\right), & t>T_{2},-l_{\epsilon}<x<l_{\epsilon} \\ N\left(t, \pm l_{\epsilon}\right) \geq 0, & t \geq T_{2} \\ N\left(T_{2}, x\right)>0, & -l_{\epsilon} \leq x \leq l_{\epsilon}\end{cases}
$$

By Proposition 3.1, we get

$$
\liminf _{t \rightarrow \infty} N(t, x)>1-\left(c_{1}+\delta\right)-\epsilon \text { uniformly on }[-L, L]
$$

In view of the arbitrariness of $L, \epsilon$ and $\delta$, we derive

$$
\liminf _{t \rightarrow \infty} N(t, x) \geq 1-c_{1} \stackrel{\text { def }}{=} \underline{N}_{1} \text { uniformly on the compact subset of } \mathbb{R} \text {. }
$$


Take $L \gg 1,0<\epsilon, \delta, \sigma \ll 1$, and $l_{\epsilon}$ be determined by Lemma 3.2 with $\beta=k_{1}, \varrho=\underline{N}_{1}-\delta, \theta=m_{1}+c_{2} \sigma$ and $\kappa=0$. Thanks to (3.9) and (3.12), we may find $T_{3}>T_{2}$ such that

$$
\frac{Z}{P+Z}<\frac{\eta}{\eta+P}<\sigma, \quad N(t, x) \geq \underline{N}_{1}-\delta \text { in }\left[T_{3}, \infty\right) \times\left[-l_{\epsilon}, l_{\epsilon}\right]
$$

Then, $P$ obeys

$$
\begin{cases}P_{t}-D_{2} P_{x x} \geq P\left(k_{1} \frac{\underline{N}_{1}-\delta}{\underline{N}_{1}-\delta+P}-m_{1}-c_{2} \sigma\right), & t>T_{3},-l_{\epsilon}<x<l_{\epsilon}, \\ P\left(t, \pm l_{\epsilon}\right) \geq 0, & t \geq T_{3}, \\ P\left(T_{3}, x\right)>0, & -l_{\epsilon} \leq x \leq l_{\epsilon} .\end{cases}
$$

Again, by using Lemma 3.2, we infer

$$
\liminf _{t \rightarrow \infty} P(t, x)>\left(\frac{k_{1}}{m_{1}+c_{2} \sigma}-1\right)\left(\underline{N}_{1}-\delta\right)-\epsilon \text { uniformly on }[-L, L] .
$$

Because of the arbitrariness of $L, \epsilon, \delta$ and $\sigma$, we check

$$
\liminf _{t \rightarrow \infty} P(t, x) \geq\left(\frac{k_{1}}{m_{1}}-1\right) \underline{N}_{1} \stackrel{\text { def }}{=} \underline{P}_{1} \text { uniformly on the compact subset of } \mathbb{R} .
$$

Step 3: The constructions of $\underline{N}_{2}$ and $\underline{P}_{2}$.

Choose $L \gg 1,0<\epsilon, \delta \ll 1$. Let $l_{\epsilon}$ be as in Proposition 3.1 with $\beta=1-c_{1} \frac{\bar{P}_{1}+\delta}{\underline{N}_{1}+\bar{P}_{1}}, \theta=1$ and $\kappa=0$. Owing to (3.12) and (3.13), there is $T_{4}>T_{3}$ such that

$$
N(t, x) \geq \underline{N}_{1}-\delta, P(t, x) \leq \bar{P}_{1}+\delta \text { in }\left[T_{4}, \infty\right) \times\left[-l_{\epsilon}, l_{\epsilon}\right] .
$$

Accordingly, $N$ satisfies

$$
\begin{cases}N_{t}-D_{1} N_{x x} \geq N\left(1-N-c_{1} \frac{\bar{P}_{1}+\delta}{\underline{N}_{1}+\bar{P}_{1}}\right), & t>T_{4},-l_{\epsilon}<x<l_{\epsilon} \\ N\left(t, \pm l_{\epsilon}\right) \geq 0, & t \geq T_{4} \\ N\left(T_{4}, x\right)>0, & -l_{\epsilon} \leq x \leq l_{\epsilon} .\end{cases}
$$

Utilizing Proposition 3.1, we deduce

$$
\liminf _{t \rightarrow \infty} N(t, x)>1-c_{1} \frac{\bar{P}_{1}+\delta}{\underline{N}_{1}+\bar{P}_{1}}-\epsilon \text { uniformly on }[-L, L] .
$$

In virtue of the arbitrariness of $L, \epsilon$ and $\delta$, we have

$$
\liminf _{t \rightarrow \infty} N(t, x) \geq 1-c_{1} \frac{\bar{P}_{1}}{\underline{N}_{1}+\bar{P}_{1}} \stackrel{\text { def }}{=} \underline{N}_{2} \text { uniformly on the compact subset of } \mathbb{R} \text {. }
$$

Select $L \gg 1,0<\epsilon, \delta, \sigma \ll 1$. Let $l_{\epsilon}$ be governed by Lemma 3.2 with $\beta=k_{1}, \varrho=N_{2}-\delta, \theta=m_{1}+c_{2} \sigma$ and $\kappa=0$. Thanks to (3.9) and (3.14), there exists $T_{5}>T_{4}$ such that

$$
\frac{Z}{P+Z}<\frac{\eta}{P+\eta}<\sigma, \quad N(t, x) \geq \underline{N}_{2}-\delta \text { in }\left[T_{5}, \infty\right) \times\left[-l_{\epsilon}, l_{\epsilon}\right] .
$$


As a consequence, $P$ obeys

$$
\begin{cases}P_{t}-D_{2} P_{x x} \geq P\left(k_{1} \frac{\underline{N}_{2}-\delta}{\underline{N_{2}-\delta+P}}-m_{1}-c_{2} \sigma\right), & t>T_{5},-l_{\epsilon}<x<l_{\epsilon}, \\ P\left(t, \pm l_{\epsilon}\right) \geq 0, & t \geq T_{5}, \\ P\left(T_{5}, x\right)>0, & -l_{\epsilon} \leq x \leq l_{\epsilon} .\end{cases}
$$

Employing Lemma 3.2, we conclude

$$
\liminf _{t \rightarrow \infty} P(t, x)>\left(\frac{k_{1}}{m_{1}+c_{2} \sigma}-1\right)\left(\underline{N}_{2}-\delta\right)-\epsilon \text { uniformly on }[-L, L]
$$

In light of the arbitrariness of $L, \epsilon, \delta$ and $\sigma$, we observe

$$
\liminf _{t \rightarrow \infty} P(t, x) \geq\left(\frac{k_{1}}{m_{1}}-1\right) \underline{N}_{2} \stackrel{\text { def }}{=} \underline{P}_{2} \text { uniformly on the compact subset of } \mathbb{R} \text {. }
$$

Step 4: The constructions of $\bar{N}_{2}, \bar{P}_{2}$.

For any given $L \gg 1$ and $0<\epsilon, \delta \ll 1$, let $l_{\epsilon}$ be determined by Proposition 3.1 with $\beta=1-c_{1} \frac{\underline{P}_{2}-\delta}{\bar{N}_{1}+\underline{P}_{2}}, \theta=1$ and $\kappa=M$. According to (3.10) and (3.15), we can find $T_{6}>T_{5}$ such that

$$
N(t, x) \leq \bar{N}_{1}+\delta, P(t, x) \geq \underline{P}_{2}-\delta \text { in }\left[T_{6}, \infty\right) \times\left[-l_{\epsilon}, l_{\epsilon}\right] .
$$

As a result, $N$ satisfies

$$
\begin{cases}N_{t}-D_{1} N_{x x} \leq N\left(1-N-c_{1} \frac{P_{2}-\delta}{\bar{N}_{1}+\underline{P}_{2}}\right), & t>T_{6},-l_{\epsilon}<x<l_{\epsilon} \\ N\left(t, \pm l_{\epsilon}\right) \leq M, & t \geq T_{6} \\ N\left(T_{6}, x\right)>0, & -l_{\epsilon} \leq x \leq l_{\epsilon}\end{cases}
$$

Using Proposition 3.1, we discover

$$
\limsup _{t \rightarrow \infty} N(t, x)<1-c_{1} \frac{\underline{P}_{2}-\delta}{\bar{N}_{1}+\underline{P}_{2}}+\epsilon \text { uniformly on }[-L, L] .
$$

In consideration of the arbitrariness of $L, \epsilon$ and $\delta$, we have

$$
\limsup _{t \rightarrow \infty} N(t, x) \leq 1-c_{1} \frac{\underline{P}_{2}}{\bar{N}_{1}+\underline{P}_{2}} \stackrel{\text { def }}{=} \bar{N}_{2} \text { uniformly on the compact subset of } \mathbb{R} \text {. }
$$

Fix $L \gg 1,0<\epsilon, \delta \ll 1$. Let $l_{\epsilon}$ be given as in Lemma 3.2 with $\beta=k_{1}, \varrho=\bar{N}_{2}+\delta, \theta=m_{1}$ and $\kappa=M$. Thanks to (3.16), there is $T_{7}>T_{6}$ such that

$$
N(t, x) \leq \bar{N}_{2}+\delta \text { in }\left[T_{7}, \infty\right) \times\left[-l_{\epsilon}, l_{\epsilon}\right] .
$$

Consequently, $P$ obeys

$$
\begin{cases}P_{t}-D_{2} P_{x x} \leq P\left(k_{1} \frac{\bar{N}_{2}+\delta}{\bar{N}_{2}+\delta+P}-m_{1}\right), & t>T_{7},-l_{\epsilon}<x<l_{\epsilon} \\ P\left(t, \pm l_{\epsilon}\right) \leq M, & t \geq T_{7} \\ P\left(T_{7}, x\right)>0, & -l_{\epsilon} \leq x \leq l_{\epsilon}\end{cases}
$$


By Lemma 3.2, we get

$$
\limsup _{t \rightarrow \infty} P(t, x)<\left(\frac{k_{1}}{m_{1}}-1\right)\left(\bar{N}_{2}+\delta\right)+\epsilon \text { uniformly on }[-L, L]
$$

In terms of the arbitrariness of $L, \epsilon$ and $\delta$, we derive

$$
\limsup _{t \rightarrow \infty} P(t, x) \leq\left(\frac{k_{1}}{m_{1}}-1\right) \bar{N}_{2} \stackrel{\text { def }}{=} \bar{P}_{2} \text { uniformly on the compact subset of } \mathbb{R} \text {. }
$$

Combining all above equalities with the conditions $k_{1}>m_{1}$ and $c_{1}<1$, we deduce that $\bar{P}_{1}, \underline{N}_{1}, \underline{P}_{1}, \underline{N}_{2}, \underline{P}_{2}$, $\bar{N}_{2}$ and $\bar{P}_{2}$ are positive constants.

Step 5: Continuing the above routine, we obtain four sequences $\left\{\bar{N}_{i}\right\},\left\{\bar{P}_{i}\right\},\left\{\underline{N}_{i}\right\}$ and $\left\{\underline{P}_{i}\right\}$, whose monotonicity is straightforward; namely, for all $i=2,3, \cdots$,

$$
\begin{aligned}
& \underline{N}_{1} \leq \cdots \leq \underline{N}_{i} \leq \cdots \leq \liminf _{t \rightarrow \infty} N(t, x) \leq \limsup _{t \rightarrow \infty} N(t, x) \leq \cdots \leq \bar{N}_{i} \leq \cdots \leq \bar{N}_{1} \\
& \underline{P}_{1} \leq \cdots \leq \underline{P}_{i} \leq \cdots \leq \liminf _{t \rightarrow \infty} P(t, x) \leq \limsup _{t \rightarrow \infty} P(t, x) \leq \cdots \leq \bar{P}_{i} \leq \cdots \leq \bar{P}_{1}
\end{aligned}
$$

uniformly on the compact subset of $\mathbb{R}$. Furthermore, these sequences can be determined by the following iterative formulas:

$$
\bar{P}_{i}=\left(\frac{k_{1}}{m_{1}}-1\right) \bar{N}_{i}, \underline{P}_{i}=\left(\frac{k_{1}}{m_{1}}-1\right) \underline{N}_{i}, \underline{N}_{i+1}=1-\frac{c_{1} \bar{P}_{i}}{\underline{N}_{i}+\bar{P}_{i}}, \bar{N}_{i+1}=1-\frac{c_{1} \underline{P}_{i+1}}{\bar{N}_{i}+\underline{P}_{i+1}}, \quad i=1,2, \cdots
$$

Because of the constant sequences $\left\{\bar{N}_{i}\right\},\left\{\bar{P}_{i}\right\}$ are monotone non-increasing and bounded, $\left\{\underline{N}_{i}\right\},\left\{\underline{P}_{i}\right\}$ are monotone non-decreasing and bounded. Hence, all of those sequences limits of exist. Let us denote their limits by $\bar{N}, \bar{P}, \underline{N}$ and $\underline{P}$, respectively. Then,

$$
\bar{P}=\left(\frac{k_{1}}{m_{1}}-1\right) \bar{N}, \quad \underline{P}=\left(\frac{k_{1}}{m_{1}}-1\right) \underline{N}, \quad \underline{N}=1-\frac{c_{1} \bar{P}}{\underline{N}+\bar{P}}, \quad \bar{N}=1-\frac{c_{1} \underline{P}}{\bar{N}+\underline{P}}
$$

This, together with the conditions $k_{1}>m_{1}$ and $c_{1}<1$, yields $\bar{N}=\underline{N}=N_{2}^{*}$ and $\bar{P}=\underline{P}=P_{2}^{*}$. Thus, from (3.18) and (3.19), we get (3.6) and (3.7).

\subsection{Spreading case: $h_{\infty}-g_{\infty}=\infty$}

For convenience of later analysis, we now present the following comparison principle which can be proved by the same way as that of Lemma 3.5 from [6] and the details will be omitted.

Lemma 3.6. (Comparison principle) Let $T \in(0, \infty), \bar{g}, \bar{h} \in C^{1}([0, T]), \bar{Z} \in C^{1,2}\left(I_{T}^{(\bar{g}, \bar{h})}\right) \cap C\left(\bar{I}_{T}^{(\bar{g}, \bar{h})}\right)$ with $I_{T}^{(\bar{g}, \bar{h})}=\left\{(t, x) \in \mathbb{R}^{2}: t \in(0, T], x \in(\bar{g}(t), \bar{h}(t))\right\}, \zeta \in C^{1}$ and obeys $\zeta(0)=0$. Assume that $(\bar{Z}, \bar{g}, \bar{h})$ satisfies

$$
\begin{cases}\bar{Z}_{t}-\bar{Z}_{x x} \geq \zeta(\bar{Z}), & 0<t \leq T, \bar{g}(t)<x<\bar{h}(t), \\ \bar{Z}(t, x)=0, & 0 \leq t \leq T, x \notin(\bar{g}(t), \bar{h}(t)), \\ \bar{g}^{\prime}(t) \leq-\mu \bar{Z}_{x}(t, \bar{g}(t)), \quad \bar{h}^{\prime}(t) \geq-\mu \bar{Z}_{x}(t, \bar{h}(t)), & 0 \leq t \leq T .\end{cases}
$$


If $\bar{g}(0) \leq-h_{0}, \bar{h}(0) \geq h_{0}$, and $\bar{Z}(0, x) \geq \phi_{3}(x)$ in $\left[-h_{0}, h_{0}\right]$, then the solution $(N, P, Z, g, h)$ of (1.4) satisfies

$$
g(t) \geq \bar{g}(t), h(t) \leq \bar{h}(t) \text { in }[0, T] ; \quad Z(t, x) \leq \bar{Z}(t, x) \text { in }[0, T] \times[g(t), h(t)] .
$$

The pair $(\bar{Z}, \bar{g}, \bar{h})$ in Lemma 3.6 is usually called an upper solution of $(Z, g, h)$. Moreover, suppose that $(\underline{Z}, \underline{g}, \underline{h})$ satisfies the conditions in Lemma 3.6 and (3.20) in which all inequalities in the obvious places are reversed, then we may obtain that $g(t) \leq g(t), h(t) \geq \underline{h}(t)$ in $[0, T]$ and $Z(t, x) \geq \underline{Z}(t, x)$ in $[0, T] \times[g(t), h(t)]$.

Next, we give the following proposition which asserts the equivalence of $h_{\infty}-g_{\infty}=\infty$ and $g_{\infty}=-\infty$, $h_{\infty}=\infty$.

Proposition 3.7. Assume that $k_{2}>m_{2}$. Let $(N, P, Z, g, h)$ be the unique global solution of (1.4). If $h_{\infty}-g_{\infty}=$ $\infty$, then $g_{\infty}=-\infty$ and $h_{\infty}=\infty$.

Proof. Suppose that $h_{\infty}<\infty$. Then the condition $h_{\infty}-g_{\infty}=\infty$ implies $g_{\infty}=-\infty$, and yet there is $T \gg 1$ such that

$$
h_{0}-g(T)>\pi \sqrt{\frac{1}{k_{2}-m_{2}}} .
$$

Hence, $Z$ satisfies

$$
\begin{cases}Z_{t}-Z_{x x} \geq-m_{2} Z, & t>T, g(T)<x<h(t), \\ Z(t, x)=0, & t \geq T, x \notin(g(T), h(t)), \\ h^{\prime}(t)=-\mu Z_{x}(t, h(t)), & t \geq T .\end{cases}
$$

Let $(\omega, \rho)$ be the unique global solution of

$$
\begin{cases}\omega_{t}-\omega_{x x}=-m_{2} \omega, & t>T, g(T)<x<\rho(t), \\ \omega(t, x)=0, & t \geq T, x \notin(g(T), \rho(t)), \\ \rho^{\prime}(t)=-\mu \omega_{x}(t, \rho(t)), & t \geq T, \\ \rho(T)=h(T), \omega(T, x)=Z(T, x), & g(T) \leq x \leq \rho(T) .\end{cases}
$$

Using the comparison principle, we observe that $h(t) \geq \rho(t)$ in $[T, \infty)$ and $Z(t, x) \geq \omega(t, x)$ in $[T, \infty) \times$ $[g(T), \rho(t)]$. According to

$$
\rho(T)-g(T)=h(T)-g(T)>h_{0}-g(T)>\pi \sqrt{\frac{1}{k_{2}-m_{2}}},
$$

by Theorem 3.2 from [21], we conclude $\lim _{t \rightarrow \infty} h(t)=\infty$, i.e., $h_{\infty}=\infty$. This result contradicts to our assumption. So, $h_{\infty}=\infty$ holds. Likewise, we may prove $g_{\infty}=-\infty$. The proof is complete.

Theorem 3.8. Assume $k_{2}>m_{2}, k_{1}>m_{1}+c_{2}$ and $c_{1}<1$ hold. Let $(N, P, Z, g, h)$ be the unique global solution of (1.4). If $h_{\infty}-g_{\infty}=\infty$, then

$$
\lim _{t \rightarrow \infty} N(t, x)=N_{3}^{*}, \quad \lim _{t \rightarrow \infty} P(t, x)=P_{3}^{*}, \quad \lim _{t \rightarrow \infty} Z(t, x)=Z_{3}^{*}
$$

uniformly in any compact subset of $\mathbb{R}$.

Proof. Take $M=\max \left\{M_{1}, M_{2}, M_{3}\right\}$ is defining as in the proof of Theorem 3.5. As before, we construct the following iteration sequences $\left\{\bar{N}_{i}\right\},\left\{\bar{P}_{i}\right\},\left\{\bar{Z}_{i}\right\},\left\{\underline{N}_{i}\right\},\left\{\underline{P}_{i}\right\}$ and $\left\{\underline{Z}_{i}\right\}$. 
Step 1: The constructions of $\bar{N}_{1}, \bar{P}_{1}$ and $\bar{Z}_{1}$.

Taking advantage of (3.10) and (3.11), we observe that

$$
\limsup _{t \rightarrow \infty} N(t, x) \leq 1 \stackrel{\text { def }}{=} \bar{N}_{1} \text { uniformly on the compact subset of } \mathbb{R}
$$

and

$$
\limsup _{t \rightarrow \infty} P(t, x) \leq \frac{k_{1}}{m_{1}}-1 \stackrel{\text { def }}{=} \bar{P}_{1} \text { uniformly on the compact subset of } \mathbb{R} \text {. }
$$

Set $L \gg 1$ and $0<\epsilon, \delta \ll 1$. Let $l_{\epsilon}$ be given as in Lemma 3.2 with $\beta=k_{2}, \varrho=\bar{P}_{1}+\delta, \theta=m_{2}$ and $\kappa=M$. According to $g_{\infty}=-\infty$ and $h_{\infty}=\infty$, there exists $T_{8}>0$ such that $g(t)<-l_{\epsilon}$ and $h(t)>l_{\epsilon}$ for $t \geq T_{8}$. In virtue of (3.23), we have

$$
P \leq \bar{P}_{1}+\delta \text { in }\left[T_{8}, \infty\right) \times\left[-l_{\epsilon}, l_{\epsilon}\right]
$$

Then, $Z$ obeys

$$
\begin{cases}Z_{t}-Z_{x x} \leq Z\left(k_{2} \frac{\bar{P}_{1}+\delta}{\bar{P}_{1}+\delta+Z}-m_{2}\right), & t>T_{8},-l_{\epsilon}<x<l_{\epsilon} \\ Z\left(t, \pm l_{\epsilon}\right) \leq M, & t \geq T_{8} \\ Z\left(T_{8}, x\right)>0, & -l_{\epsilon} \leq x \leq l_{\epsilon}\end{cases}
$$

Applying Lemma 3.2, we derive

$$
\limsup _{t \rightarrow \infty} Z(t, x)<\left(\frac{k_{2}}{m_{2}}-1\right)\left(\bar{P}_{1}+\delta\right)+\epsilon \text { uniformly on }[-L, L] .
$$

Because of the arbitrariness of $L, \epsilon$ and $\delta$, we check

$$
\begin{aligned}
& \limsup _{t \rightarrow \infty} Z(t, x) \leq\left(\frac{k_{2}}{m_{2}}-1\right) \bar{P}_{1}=\left(\frac{k_{2}}{m_{2}}-1\right)\left(\frac{k_{1}}{m_{1}}-1\right) \\
& \stackrel{\text { def }}{=} \bar{Z}_{1} \text { uniformly on the compact subset of } \mathbb{R} .
\end{aligned}
$$

Step 2: The constructions of $\underline{N}_{1}, \underline{P}_{1}$ and $\underline{Z}_{1}$.

Choose $L \gg 1,0<\epsilon, \delta \ll 1$. Let $l_{\epsilon}$ be determined by Proposition 3.1 with $\beta=1-c_{1}-\delta, \theta=1$ and $\kappa=0$. Thus, we may find $T_{9}>T_{8}$ such that $N$ satisfies

$$
\begin{cases}N_{t}-D_{1} N_{x x} \geq N\left(1-N-c_{1}-\delta\right), & t>T_{9},-l_{\epsilon}<x<l_{\epsilon} \\ N\left(t, \pm l_{\epsilon}\right) \geq 0, & t \geq T_{9} \\ N\left(T_{9}, x\right)>0, & -l_{\epsilon} \leq x \leq l_{\epsilon}\end{cases}
$$

By Proposition 3.1, we deduce

$$
\liminf _{t \rightarrow \infty} N(t, x)>1-c_{1}-\delta-\epsilon \text { uniformly on }[-L, L] .
$$

In consideration of the arbitrariness of $L, \epsilon$ and $\delta$, we discover

$$
\liminf _{t \rightarrow \infty} N(t, x) \geq 1-c_{1} \stackrel{\text { def }}{=} \underline{N}_{1} \text { uniformly on the compact subset of } \mathbb{R} \text {. }
$$


Take $L \gg 1,0<\epsilon, \delta, \eta \ll 1$. Let $l_{\epsilon}$ be governed by Lemma 3.2 with $\beta=k_{1}, \varrho=\underline{N}_{1}-\delta, \theta=m_{1}+c_{2}+\eta$ and $\kappa=0$. Thanks to $(3.25)$, there is $T_{10}>T_{9}$ such that

$$
N(t, x) \geq \underline{N}_{1}-\delta \text { in }\left[T_{10}, \infty\right) \times\left[-l_{\epsilon}, l_{\epsilon}\right] .
$$

Consequently, $P$ obeys

$$
\begin{cases}P_{t}-D_{2} P_{x x} \geq P\left(k_{1} \frac{\underline{N}_{1}-\delta}{\underline{N}_{1}-\delta+P}-m_{1}-c_{2}-\eta\right), & t>T_{10},-l_{\epsilon}<x<l_{\epsilon}, \\ P\left(t, \pm l_{\epsilon}\right) \geq 0, & t \geq T_{10}, \\ P\left(T_{10}, x\right)>0, & -l_{\epsilon} \leq x \leq l_{\epsilon} .\end{cases}
$$

Arguing as the same as step 1 ,

$$
\begin{aligned}
& \liminf _{t \rightarrow \infty} P(t, x) \geq\left(\frac{k_{1}}{m_{1}+c_{2}}-1\right) \underline{N}_{1}=\left(\frac{k_{1}}{m_{1}+c_{2}}-1\right)\left(1-c_{1}\right) \\
& \stackrel{\text { def }}{=} \underline{P}_{1} \text { uniformly on the compact subset of } \mathbb{R} .
\end{aligned}
$$

Fix $L \gg 1$ and $0<\epsilon, \delta \ll 1$. Let $l_{\epsilon}$ be given as in Lemma 3.2 with $\beta=k_{2}, \varrho=\underline{P}_{1}-\delta, \theta=m_{2}$ and $\kappa=0$. Owing to $g_{\infty}=-\infty$ and $h_{\infty}=\infty$, there exists $T_{11}>T_{10}$ such that $g(t)<-l_{\epsilon}$ and $h(t)>l_{\epsilon}$ for $t \geq T_{11}$. In terms of (3.26), we get

$$
P(t, x) \geq \underline{P}_{1}-\delta \text { in }\left[T_{11}, \infty\right) \times\left[-l_{\epsilon}, l_{\epsilon}\right]
$$

Hence, $Z$ satisfies

$$
\begin{cases}Z_{t}-Z_{x x} \geq Z\left(k_{2} \frac{\underline{P}_{1}-\delta}{\underline{P}_{1}-\delta+Z}-m_{2}\right), & t>T_{11},-l_{\epsilon}<x<l_{\epsilon} \\ Z\left(t, \pm l_{\epsilon}\right) \geq 0, & t \geq T_{11} \\ Z\left(T_{11}, x\right)>0, & -l_{\epsilon} \leq x \leq l_{\epsilon}\end{cases}
$$

Similar to step 1 ,

$$
\begin{aligned}
& \liminf _{t \rightarrow \infty} Z(t, x)>\left(\frac{k_{2}}{m_{2}}-1\right) \underline{P}_{1}=\left(\frac{k_{2}}{m_{2}}-1\right)\left(\frac{k_{1}}{m_{1}+c_{2}}-1\right)\left(1-c_{1}\right) \\
& \stackrel{\text { def }}{=} \underline{Z}_{1} \text { uniformly on the compact subset of } \mathbb{R} .
\end{aligned}
$$

Step 3: The constructions of $\underline{N}_{2}, \underline{P}_{2}, \underline{Z}_{2}$.

For any given $L \gg 1$ and $0<\epsilon, \delta \ll 1$, let $l_{\epsilon}$ be determined by Proposition 3.1 with $\beta=1-c_{1} \frac{\bar{P}_{1}+\delta}{\underline{N}_{1}+\bar{P}_{1}}, \theta=1$ and $\kappa=0$. According to (3.23) and (3.25), we can find $T_{12}>T_{11}$ such that

$$
P(t, x) \leq \bar{P}_{1}+\delta, N(t, x) \geq \underline{N}_{1}-\delta \text { in }\left[T_{12}, \infty\right) \times\left[-l_{\epsilon}, l_{\epsilon}\right]
$$

As a result, $N$ obeys

$$
\begin{cases}N_{t}-D_{1} N_{x x} \geq N\left(1-N-c_{1} \frac{\bar{P}_{1}+\delta}{\underline{N}_{1}+\bar{P}_{1}}\right), & t>T_{12},-l_{\epsilon}<x<l_{\epsilon} \\ N\left(t, \pm l_{\epsilon}\right) \geq 0, & t \geq T_{12}, \\ N\left(T_{12}, x\right)>0, & -l_{\epsilon} \leq x \leq l_{\epsilon} .\end{cases}
$$


By the same arguments as step 2, we infer

$$
\begin{aligned}
& \liminf _{t \rightarrow \infty} N(t, x) \geq 1-c_{1} \frac{\bar{P}_{1}}{\underline{N}_{1}+\bar{P}_{1}}=k_{1} \frac{1-c_{1}}{k_{1}-c_{1} m_{1}} \\
& \stackrel{\text { def }}{=} \underline{N}_{2} \text { uniformly on the compact subset of } \mathbb{R} .
\end{aligned}
$$

Select $L \gg 1,0<\epsilon, \delta, \eta \ll 1$. Let $l_{\epsilon}$ be governed by Lemma 3.2 with $\beta=k_{1}, \theta=m_{1}+c_{2}+\eta$ and $\kappa=0$. Thanks to (3.28), there is $T_{13}>T_{12}$ such that

$$
N(t, x) \geq \underline{N}_{2}-\delta \text { in }\left[T_{13}, \infty\right) \times\left[-l_{\epsilon}, l_{\epsilon}\right] .
$$

As a consequence, $P$ satisfies

$$
\begin{cases}P_{t}-D_{2} P_{x x} \geq P\left(k_{1} \frac{\underline{N}_{2}-\delta}{\underline{N}_{2}-\delta+P}-m_{1}-c_{2}-\eta\right), & t>T_{13},-l_{\epsilon}<x<l_{\epsilon}, \\ P\left(t, \pm l_{\epsilon}\right) \geq 0, & t \geq T_{13}, \\ P\left(T_{13}, x\right)>0, & -l_{\epsilon} \leq x \leq l_{\epsilon} .\end{cases}
$$

Arguing as the same as step 1,

$$
\begin{aligned}
& \liminf _{t \rightarrow \infty} P(t, x) \geq\left(\frac{k_{1}}{m_{1}+c_{2}}-1\right) \underline{N}_{2}=k_{1}\left(\frac{k_{1}}{m_{1}+c_{2}}-1\right)\left(\frac{1-c_{1}}{k_{1}-c_{1} m_{1}}\right) \\
& \stackrel{\text { def }}{=} \underline{P}_{2} \text { uniformly on the compact subset of } \mathbb{R} .
\end{aligned}
$$

Let $L \gg 1,0<\epsilon, \delta \ll 1$, and $l_{\epsilon}$ be given by Lemma 3.2 with $\beta=k_{2}, \varrho=\underline{P}_{2}-\delta, \theta=m_{2}$ and $\kappa=0$. Owing to $g_{\infty}=-\infty$ and $h_{\infty}=\infty$, there exists $T_{14}>T_{13}$ such that $g(t)<-l_{\epsilon}$ and $h(t)>l_{\epsilon}$ for $t \geq T_{14}$. By (3.29), we have

$$
P(t, x) \geq \underline{P}_{2}-\delta \text { in }\left[T_{14}, \infty\right) \times\left[-l_{\epsilon}, l_{\epsilon}\right]
$$

Accordingly, $Z$ obeys

$$
\begin{cases}Z_{t}-Z_{x x} \geq Z\left(k_{2} \frac{P_{2}-\delta}{\underline{P}_{2}-\delta+Z}-m_{2}\right), & t>T_{14},-l_{\epsilon}<x<l_{\epsilon} \\ Z\left(t, \pm l_{\epsilon}\right) \geq 0, & t \geq T_{14} \\ Z\left(T_{14}, x\right)>0, & -l_{\epsilon} \leq x \leq l_{\epsilon}\end{cases}
$$

Similar to step 1 ,

$$
\begin{aligned}
& \liminf _{t \rightarrow \infty} Z(t, x) \geq\left(\frac{k_{2}}{m_{2}}-1\right) \underline{P}_{2}=k_{1}\left(\frac{k_{2}}{m_{2}}-1\right)\left(\frac{k_{1}}{m_{1}+c_{2}}-1\right)\left(\frac{1-c_{1}}{k_{1}-c_{1} m_{1}}\right) \\
& \stackrel{\text { def }}{=} \underline{Z}_{2} \text { uniformly on the compact subset of } \mathbb{R} .
\end{aligned}
$$

Step 4: The constructions of $\bar{N}_{2}, \bar{P}_{2}, \bar{Z}_{2}$.

Take $L \gg 1$ and $0<\epsilon, \delta \ll 1$. Let $l_{\epsilon}$ be determined by Proposition 3.1 with $\beta=1-c_{1} \frac{\underline{P}_{2}-\delta}{\bar{N}_{1}+\underline{P}_{2}}, \theta=1$ and $\kappa=M$. According to (3.22) and (3.29), we may find $T_{15}>T_{14}$ such that

$$
N(t, x) \leq \bar{N}_{1}+\delta, P(t, x) \geq \underline{P}_{2}-\delta \text { in }\left[T_{15}, \infty\right) \times\left[-l_{\epsilon}, l_{\epsilon}\right]
$$


As a result, $N$ obeys

$$
\begin{cases}N_{t}-D_{1} N_{x x} \leq N\left(1-N-c_{1} \frac{\underline{P}_{2}-\delta}{\bar{N}_{1}+\underline{P}_{2}}\right), & t>T_{15},-l_{\epsilon}<x<l_{\epsilon} \\ N\left(t, \pm l_{\epsilon}\right) \leq M, & t \geq T_{15} \\ N\left(T_{15}, x\right)>0, & -l_{\epsilon} \leq x \leq l_{\epsilon} .\end{cases}
$$

By the same argument as step 2, we conclude

$$
\limsup _{t \rightarrow \infty} N(t, x) \leq 1-c_{1} \frac{\underline{P}_{2}}{\bar{N}_{1}+\underline{P}_{2}} \stackrel{\text { def }}{=} \bar{N}_{2} \text { uniformly on the compact subset of } \mathbb{R} .
$$

Set $L \gg 1,0<\epsilon, \delta \ll 1$. Let $l_{\epsilon}$ be governed by Lemma 3.2 with $\beta=k_{1}, \varrho=\bar{N}_{2}+\delta, \theta=m_{1}$ and $\kappa=M$. Thanks to (3.31), there is $T_{16}>T_{15}$ such that

$$
N(t, x) \leq \bar{N}_{2}+\delta \text { in }\left[T_{16}, \infty\right) \times\left[-l_{\epsilon}, l_{\epsilon}\right]
$$

Therefore, $P$ satisfies

$$
\begin{cases}P_{t}-D_{2} P_{x x} \leq P\left(k_{1} \frac{\bar{N}_{2}+\delta}{\bar{N}_{2}+\delta+P}-m_{1}\right), & t>T_{16},-l_{\epsilon}<x<l_{\epsilon}, \\ P\left(t, \pm l_{\epsilon}\right) \leq M, & t \geq T_{16}, \\ P\left(T_{16}, x\right)>0, & -l_{\epsilon} \leq x \leq l_{\epsilon} .\end{cases}
$$

Arguing as the same as step 1,

$$
\limsup _{t \rightarrow \infty} P(t, x) \leq\left(\frac{k_{1}}{m_{1}}-1\right) \bar{N}_{2} \stackrel{\text { def }}{=} \bar{P}_{2} \text { uniformly on the compact subset of } \mathbb{R} \text {. }
$$

Fix $L \gg 1,0<\epsilon, \delta \ll 1$. Let $l_{\epsilon}$ be given as in Lemma 3.2 with $\beta=k_{2}, \varrho=\bar{P}_{2}+\delta, \theta=m_{2}$ and $\kappa=M$. Owing to $g_{\infty}=-\infty$ and $h_{\infty}=\infty$, there exists $T_{17}>T_{16}$ such that $g(t)<-l_{\epsilon}$ and $h(t)>l_{\epsilon}$ for $t \geq T_{17}$. In view of (3.32), we observe

$$
P(t, x) \leq \bar{P}_{2}+\delta \text { in }\left[T_{17}, \infty\right) \times\left[-l_{\epsilon}, l_{\epsilon}\right]
$$

Hence, $Z$ obeys

$$
\begin{cases}Z_{t}-Z_{x x} \leq Z\left(k_{2} \frac{\bar{P}_{2}+\delta}{\bar{P}_{2}+\delta+Z}-m_{2}\right), & t>T_{17},-l_{\epsilon}<x<l_{\epsilon} \\ Z\left(t, \pm l_{\epsilon}\right) \leq M, & t \geq T_{17} \\ Z\left(T_{17}, x\right)>0, & -l_{\epsilon} \leq x \leq l_{\epsilon} .\end{cases}
$$

Similar to step 1,

$$
\limsup _{t \rightarrow \infty} Z(t, x) \leq\left(\frac{k_{2}}{m_{2}}-1\right) \bar{P}_{2} \stackrel{\text { def }}{=} \bar{Z}_{2} \text { uniformly on the compact subset of } \mathbb{R} \text {. }
$$

Consequently, from $k_{2}>m_{2}, k_{1}>m_{1}+c_{2}$ and $c_{1}<1$, we deduce that $\bar{P}_{1}, \bar{Z}_{1}, \underline{N}_{1}, \underline{P}_{1}, \underline{Z}_{1}, \underline{N}_{2}, \underline{P}_{2}, \underline{Z}_{2}, \bar{N}_{2}$, $\bar{P}_{2}$ and $\bar{Z}_{2}$ are positive constants. 
Step 5: Repeating the above procedure, we can find six sequences $\left\{\bar{N}_{i}\right\},\left\{\bar{P}_{i}\right\},\left\{\bar{Z}_{i}\right\},\left\{\underline{N}_{i}\right\},\left\{\underline{P}_{i}\right\}$ and $\left\{\underline{Z}_{i}\right\}$, whose monotonicity is straightforward; namely, for all $i=2,3, \cdots$,

$$
\begin{aligned}
& \underline{N}_{1} \leq \cdots \leq \underline{N}_{i} \leq \cdots \leq \liminf _{t \rightarrow \infty} N(t, x) \leq \limsup _{t \rightarrow \infty} N(t, x) \leq \cdots \leq \bar{N}_{i} \leq \cdots \leq \bar{N}_{1} \\
& \underline{P}_{1} \leq \cdots \leq \underline{P}_{i} \leq \cdots \leq \liminf _{t \rightarrow \infty} P(t, x) \leq \limsup _{t \rightarrow \infty} P(t, x) \leq \cdots \leq \bar{P}_{i} \leq \cdots \leq \bar{P}_{1} \\
& \underline{Z}_{1} \leq \cdots \leq \underline{Z}_{i} \leq \cdots \leq \liminf _{t \rightarrow \infty} Z(t, x) \leq \limsup _{t \rightarrow \infty} Z(t, x) \leq \cdots \leq \bar{Z}_{i} \leq \cdots \leq \bar{Z}_{1}
\end{aligned}
$$

uniformly on the compact subset of $\mathbb{R}$. Moreover, these sequences can be governed by the following iterative formulas:

$$
\begin{aligned}
& \bar{P}_{i}=\left(\frac{k_{1}}{m_{1}}-1\right) \bar{N}_{i}, \quad \bar{Z}_{i}=\left(\frac{k_{2}}{m_{2}}-1\right) \bar{P}_{i}, \quad \bar{N}_{i+1}=1-\frac{c_{1} \underline{P}_{i+1}}{\bar{N}_{i}+\underline{P}_{i+1}} \\
& \underline{P}_{i}=\left(\frac{k_{1}}{m_{1}+c_{2}}-1\right) \underline{N}_{i}, \quad \underline{Z}_{i}=\left(\frac{k_{2}}{m_{2}}-1\right) \underline{P}_{i}, \quad \underline{N_{i+1}}=1-\frac{c_{1} \bar{P}_{i}}{\underline{N}_{i}+\bar{P}_{i}} \quad \text { for } i=1,2, \cdots .
\end{aligned}
$$

Since the constant sequences $\left\{\bar{N}_{i}\right\},\left\{\bar{P}_{i}\right\},\left\{\bar{Z}_{i}\right\}$ are monotone non-increasing and bounded, $\left\{\underline{N}_{i}\right\},\left\{\underline{P}_{i}\right\},\left\{\underline{Z}_{i}\right\}$ are monotone non-decreasing and bounded, the limits of these sequences exist. Denote their limits by $\bar{N}, \bar{P}, \bar{Z}$, $\underline{N}, \underline{P}$ and $\underline{Z}$, respectively. Thus,

$$
\left\{\begin{array}{lll}
\bar{P}=\left(\frac{k_{1}}{m_{1}}-1\right) \bar{N}, & \bar{Z}=\left(\frac{k_{2}}{m_{2}}-1\right) \bar{P}, & \bar{N}=1-\frac{c_{1} \underline{P}}{\bar{N}+\underline{P}} \\
\underline{P}=\left(\frac{k_{1}}{m_{1}+c_{2}}-1\right) \underline{N}, & \underline{Z}=\left(\frac{k_{2}}{m_{2}}-1\right) \underline{P}, & \underline{N}=1-\frac{c_{1}}{\underline{N}+\bar{P}}
\end{array}\right.
$$

With the assumptions, we derive $\bar{N}=\underline{N}=N_{3}^{*}, \bar{P}=\underline{P}=P_{3}^{*}$ and $\bar{Z}=\underline{Z}=Z_{3}^{*}$. Therefore, from (3.34), (3.35), and (3.36), it follows that (3.21).

\section{The CRITERIA GOVERNING SPREADING AND VANISHING}

In this section, we focus on the criteria governing spreading and vanishing. We first give a necessary condition for vanishing.

Theorem 4.1. Suppose that $k_{1}>m_{1}, k_{2}>m_{2}$ and $c_{1}<1$. Let $(N, P, Z, g, h)$ be the unique global solution of (1.4). If $h_{\infty}-g_{\infty}<\infty$, then

$$
h_{\infty}-g_{\infty} \leq \Lambda \stackrel{\text { def }}{=} \pi \sqrt{\frac{1}{k_{2}-m_{2}}} .
$$

Proof. In view of Theorem 3.5, the condition $h_{\infty}-g_{\infty}<\infty$ implies that $\lim _{t \rightarrow \infty}\|Z(t, x)\|_{C([g(t), h(t)])}=0$, $\lim _{t \rightarrow \infty} N(t, x)=N_{2}^{*}$ and $\lim _{t \rightarrow \infty} P(t, x)=P_{2}^{*}$ uniformly in any compact subset of $\mathbb{R}$. Suppose that $h_{\infty}-g_{\infty}>\Lambda$. For any given $0<\delta \ll 1$, there exists $T \gg 1$ such that

$$
\begin{gathered}
P(t, x) \geq P_{2}^{*}-\delta \text { in }[T, \infty) \times\left[g_{\infty}, h_{\infty}\right], \\
h(T)-g(T)>\max \left\{2 h_{0}, \Lambda\right\} .
\end{gathered}
$$


Let $\omega(t, x)$ be the solution of the following initial-boundary value problem

$$
\begin{cases}\omega_{t}-\omega_{x x}=\omega\left(k_{2} \frac{P_{2}^{*}-\delta}{P_{2}^{*}-\delta+\omega}-m_{2}\right), & t>T, g(T)<x<h(T), \\ \omega(t, g(T))=\omega(t, h(T))=0, & t \geq T, \\ \omega(T, x)=Z(T, x), & g(T) \leq x \leq h(T)\end{cases}
$$

Then, by using the comparison principle, we deduce $\omega(t, x) \leq Z(t, x)$ in $[T, \infty) \times[g(T), h(T)]$. Due to $h(T)-$ $g(T)>\Lambda$, one can easily see that $\omega(t, x) \rightarrow \vartheta(x)$ as $t \rightarrow \infty$ uniformly in the compact subset $(g(T), h(T))$, where $\vartheta(x)$ is the unique positive solution of

$$
\left\{\begin{array}{l}
\vartheta_{x x}+\vartheta\left(k_{2} \frac{P_{2}^{*}-\delta}{P_{2}^{*}-\delta+\vartheta}-m_{2}\right)=0, \quad g(T)<x<h(T), \\
\vartheta(g(T))=\vartheta(h(T))=0 .
\end{array}\right.
$$

Consequently,

$$
\liminf _{t \rightarrow \infty} Z(t, x) \geq \lim _{t \rightarrow \infty} \omega(t, x)=\vartheta(x)>0 \text { in }[g(T), h(T)]
$$

which is in contradiction with (3.8). So, (4.1) holds.

Remark 4.2. For all $\mu>0$, if $k_{2}>m_{2}$, then $h_{0} \geq \frac{\Lambda}{2}$ implies that $g_{\infty}=-\infty$ and $h_{\infty}=\infty$ by Proposition 3.7 and Theorem 4.1.

We now pay close attention to the case $h_{0}<\frac{\Lambda}{2}$.

Lemma 4.3. Assume that $k_{1}>m_{1}$ and $k_{2}>m_{2}$. Let $(N, P, Z, g, h)$ be the unique global solution of (1.4). If $h_{0}<\frac{\Lambda}{2}$, then there exists $\mu^{*}>0$ depending on $\left(\phi_{1}, \phi_{2}, \phi_{3}, h_{0}\right)$ such that $g_{\infty}=-\infty$ and $h_{\infty}=\infty$ if $\mu \geq \mu^{*}$.

Proof. The proof is inspired by Lemma 3.6 from [19]. Take $M=\max \left\{M_{1}, M_{2}, M_{3}\right\}$ is defining as in the proof of Theorem 3.5. Observing the third equation of (1.4), for all $P, Z \in[0, M]$, we may find a positive constant $\delta^{*}$ such that

$$
Z_{t}-Z_{x x}=Z\left(k_{2} \frac{P}{P+Z}-m_{2}\right) \geq-\delta^{*} Z
$$

Consider the following auxiliary free boundary problem

$$
\begin{cases}\omega_{t}-\omega_{x x}=-\delta^{*} \omega, & t>0, \rho_{1}(t)<x<\rho_{2}(t), \\ \omega(t, x)=0, & t \geq 0, x \notin\left(\rho_{1}(t), \rho_{2}(t)\right) \\ \rho_{1}(t)=-\mu \omega_{x}\left(t, \rho_{1}(t)\right), \quad \rho_{2}(t)=-\mu \omega_{x}\left(t, \rho_{2}(t)\right), & t \geq 0, \\ \rho_{1}(0)=-h_{0}, \rho_{2}(0)=h_{0}, \omega(0, x)=\phi_{3}(x), & -h_{0} \leq x \leq h_{0}\end{cases}
$$

Arguing as in the proof of the existence and uniqueness of the global positive solution to (1.4), we deduce that (4.2) also admits a unique positive solution $\left(\omega, \rho_{1}, \rho_{2}\right)$ defined for all $t>0$. In addition, $\omega$ is bounded above by some positive constants and $\rho_{1}^{\prime}(t)<0<\rho_{2}^{\prime}(t)$ for all $t>0$. Now we write $\left(Z^{\mu}, g^{\mu}, h^{\mu}\right)$ and $\left(\omega^{\mu}, \rho_{1}^{\mu}, \rho_{2}^{\mu}\right)$ in place of $(Z, g, h)$ and $\left(\omega, \rho_{1}, \rho_{2}\right)$ to clarify the dependence of the solutions on the parameter $\mu$.

Applying the comparison principle, we derive

$$
Z^{\mu}(t, x) \geq \omega^{\mu}(t, x), g^{\mu}(t) \leq \rho_{1}^{\mu}(t), h^{\mu}(t) \geq \rho_{2}^{\mu}(t), \quad \forall t \geq 0, x \in\left[\rho_{1}^{\mu}(t), \rho_{2}^{\mu}(t)\right] .
$$


Then there exists a constant $\mu^{*}>0$ such that $\rho_{1}^{\mu}(2) \leq-\Lambda$ and $\rho_{2}^{\mu}(2) \geq \Lambda$ for all $\mu \geq \mu^{*}$ and $t>0$ as in the proof of Lemma 3.2 in [27]. Hence,

$$
g_{\infty}=\lim _{t \rightarrow \infty} g^{\mu}(t)<g^{\mu}(2) \leq \rho_{1}^{\mu}(2) \leq-\Lambda, \quad h_{\infty}=\lim _{t \rightarrow \infty} h^{\mu}(t)>h^{\mu}(2) \geq \rho_{2}^{\mu}(2) \geq \Lambda, \quad \forall \mu \geq \mu^{*},
$$

which is equivalent to $h_{\infty}-g_{\infty}>2 \Lambda$. With Theorem 4.1, it implies $h_{\infty}-g_{\infty}=\infty$. Therefore, we deduce that $g_{\infty}=-\infty$ and $h_{\infty}=\infty$ from Proposition 3.7. This complete the proof.

Lemma 4.4. Suppose that $k_{1}>m_{1}$ and $k_{2}>m_{2}$. Let $(N, P, Z, g, h)$ be the unique global solution of (1.4). If $h_{0}<\frac{\Lambda}{2}$, then there exists $\mu_{*}>0$, depending on $\left(\phi_{1}, \phi_{2}, \phi_{3}, h_{0}\right)$ such that $h_{\infty}-g_{\infty} \leq \Lambda$ when $\mu \leq \mu_{*}$.

Proof. We first observe that $\lambda_{1}=\frac{\pi^{2}}{4 h_{0}^{2}}$ and $\varphi(x)=\sin \frac{\pi\left(x+h_{0}\right)}{2 h_{0}}$ are the principal eigenvalue and the corresponding positive eigenfunction of the problem

$$
\left\{\begin{array}{l}
-\varphi_{x x}=\lambda \varphi, \quad-h_{0}<x<h_{0} \\
\varphi\left( \pm h_{0}\right)=0,
\end{array}\right.
$$

and there exists $\gamma>0$ such that

$$
x \varphi^{\prime}(x) \leq \gamma \varphi(x), \quad \forall x \in\left[-h_{0}, h_{0}\right] .
$$

The condition $h_{0}<\frac{\Lambda}{2}$ indicates $\lambda_{1}>k_{2}-m_{2}$. Motivated by Wang [21, 22], we define

$$
\begin{aligned}
& f(t)=1+2 \delta-\delta e^{-\eta t}, \quad s(t)=h_{0} f(t), \quad t \geq 0 \\
& W(t, x)=M e^{-\eta t} \varphi\left(\frac{x}{f(t)}\right), \quad t \geq 0,-s(t) \leq x \leq s(t)
\end{aligned}
$$

where $0<\delta, \eta<1$ and $M>0$ will be selected later. Denote $y=\frac{x}{f(t)}$. Making use of (4.3) and $\lambda_{1}>k_{2}-m_{2}$, some straightforward calculations show that

$$
\begin{aligned}
& W_{t}-W_{x x}-W\left(k_{2}-m_{2}\right) \\
& =M e^{-\eta t}\left[-\eta \varphi-\varphi^{\prime} \frac{x f^{\prime}}{f^{2}}-\frac{\varphi^{\prime \prime}}{f^{2}}-\varphi\left(k_{2}-m_{2}\right)\right] \\
& \geq W\left[-\eta-\kappa \eta+\frac{\lambda_{1}}{f^{2}}-\left(k_{2}-m_{2}\right)\right] \geq 0, \quad \forall t>0,-s(t)<x<s(t)
\end{aligned}
$$

provided $0<\delta, \eta \ll 1$. Furthermore, we choose sufficiently large $M$ such that

$$
W(0, x)=M \varphi\left(\frac{x}{1+\delta}\right) \geq \phi_{3}(x), \quad \forall x \in\left[-h_{0}, h_{0}\right] .
$$

Additionally, we discover

$$
s^{\prime}(t)=h_{0} \delta \eta e^{-\eta t}, W(t, \pm s(t))=0, W_{x}(t, \pm s(t))=\frac{M e^{-\eta t}}{f(t)} \varphi^{\prime}\left( \pm h_{0}\right) .
$$

Recalling $\varphi^{\prime}\left(-h_{0}\right)>0$ and $\varphi^{\prime}\left(h_{0}\right)<0$, there is a constant $0<\mu_{*} \ll 1$ such that

$$
-h_{0} \delta \eta \leq-\mu \frac{M}{f(t)} \varphi^{\prime}\left(-h_{0}\right), \quad h_{0} \delta \eta \geq-\mu \frac{M}{f(t)} \varphi^{\prime}\left(h_{0}\right), \quad \forall t \geq 0
$$


for all $0<\mu \leq \mu_{*}$. Therefore,

$$
-s^{\prime}(t) \leq-\mu W_{x}(t,-s(t)), \quad s^{\prime}(t) \geq-\mu W_{x}(t, s(t)), \quad \forall t \geq 0 .
$$

On the other hand, $Z(t, x)$ satisfies

$$
Z_{t}-Z_{x x}-Z\left(k_{2}-m_{2}\right)<0, \quad \forall t>0, g(t)<x<h(t) .
$$

Thus, we conclude from Lemma 3.6 that

$$
g(t) \geq-s(t), \quad h(t) \leq s(t), \quad \forall t \geq 0 .
$$

This implies that

$$
g_{\infty} \geq \lim _{t \rightarrow \infty}(-s(t))=-h_{0}(1+2 \delta)>-\frac{\Lambda}{2}>-\infty, \quad h_{\infty} \leq \lim _{t \rightarrow \infty} s(t)=h_{0}(1+2 \delta)<\frac{\Lambda}{2}<\infty .
$$

So, $h_{\infty}-g_{\infty} \leq \Lambda$. The proof is finished.

Note that $\mu_{*}<\mu^{*}$. Since $\mu_{*} \geq \mu^{*}$ implies $\mu \geq \mu^{*}$ for $\mu \in\left[\mu^{*}, \mu_{*}\right]$, we deduce using Lemma 4.3 that $h_{\infty}-g_{\infty}=$ $\infty$. In addition, $\mu \leq \mu_{*}$ together with Lemma 4.4 , yields $h_{\infty}-g_{\infty} \leq \Lambda$. This is a contradiction. Hence, the following theorem can be obtained.

Theorem 4.5. Assume that $k_{1}>m_{1}$ and $k_{2}>m_{2}$. Let $(N, P, Z, g, h)$ be the unique global solution of (1.4). If $h_{0}<\frac{\Lambda}{2}$, then there exist $\mu^{*}>\mu_{*}>0$, depending on $\left(\phi_{1}, \phi_{2}, \phi_{3}, h_{0}\right)$ such that $g_{\infty}=-\infty$ and $h_{\infty}=\infty$ when $\mu \geq \mu^{*}$, and $h_{\infty}-g_{\infty} \leq \Lambda$ when $\mu \leq \mu_{*}$.

\section{NUMERICAL SIMULATIONS}

In this section, two numerical examples are carried out by Matlab to support our theoretical results. To be computable, we fix some coefficients and initial functions as follows:

$$
\begin{gathered}
D_{1}=0.5, D_{2}=0.8, c_{1}=0.838, c_{2}=0.52, k_{1}=1.5, k_{2}=2, m_{1}=0.61, m_{2}=1.05 ; \\
\phi_{1}(x)=e^{-\frac{x^{2}}{10}}, \quad \phi_{2}(x)=0.8 e^{-\frac{x^{2}}{10}}, \quad \phi_{3}(x)=0.4 \cos \left(\frac{\pi x}{2 h_{0}}\right) .
\end{gathered}
$$

It clearly follows from the above parameters that $k_{1}>m_{1}, k_{2}>m_{2}, k_{1}>m_{1}+c_{2}$ and $c_{1}<1$. This means that the conditions are maintained in Theorems 3.5 and 3.8. Then, problem (1.4) admits the semi-trivial steady state $E_{2}=\left(N_{2}^{*}, P_{2}^{*}, 0\right) \approx(0.503,0.734,0)$. Combining with the formula

$$
\mathcal{A}=\frac{k_{1}}{c_{2} \frac{k_{2}-m_{2}}{k_{2}}+m_{1}}=\frac{1.5}{0.52 \times \frac{2-1.05}{2}+0.61} \approx 1.75>1,
$$

we deduce that problem (1.4) has the unique positive constant steady state $E_{3}=\left(N_{3}^{*}, P_{3}^{*}, Z_{3}^{*}\right) \approx$ (0.641, 0.481, 0.435).

Now, we assume that the initial habitat $h_{0}$ and expanding capability $\mu$ are both changeable and design the following experiments: 


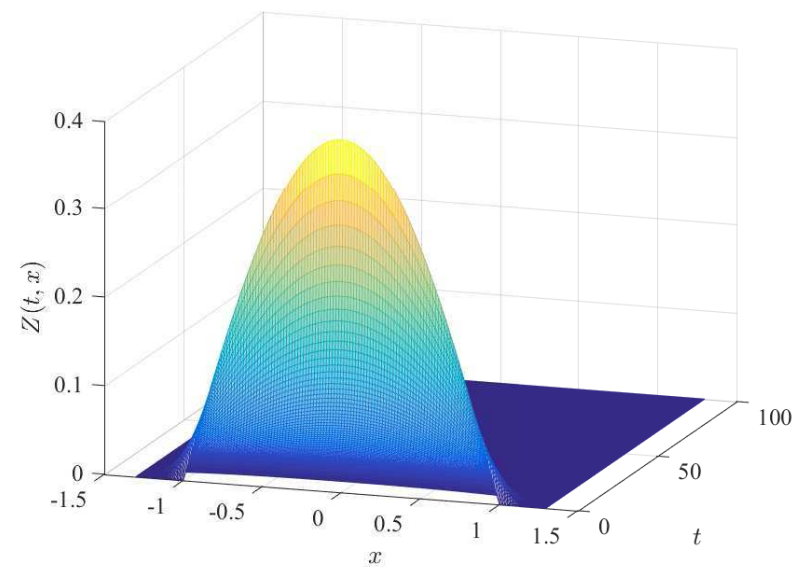

(a) vanishing case $\mu=0.6$

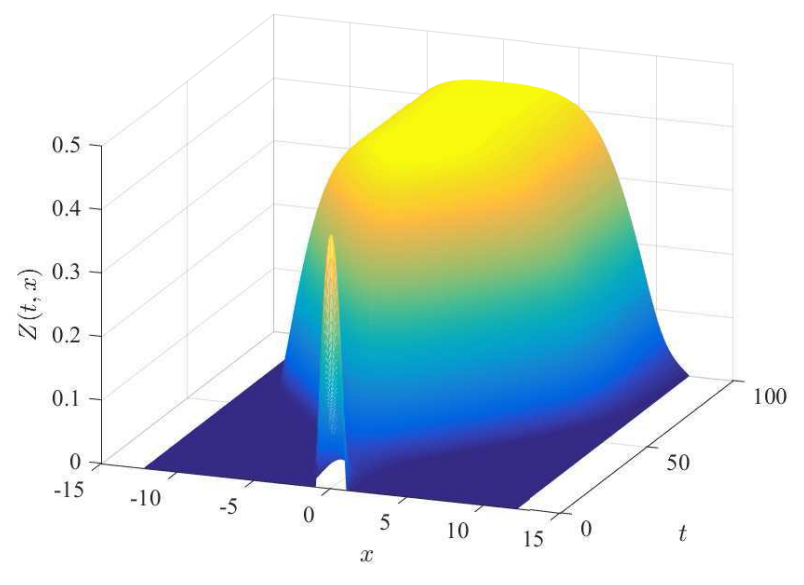

(b) spreading case $\mu=1$

Figure 1. Evolution of $(Z, g, h)$ with different expanding capabilities $\mu=0.6$ and $\mu=1$.

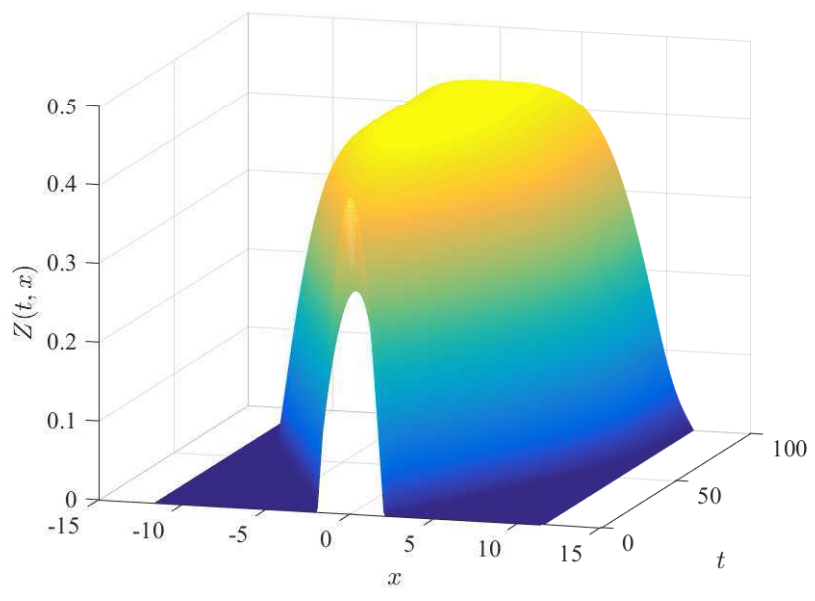

Figure 2. Evolution of $(Z, g, h)$ with expanding capability $\mu=0.6$.

Example 5.1. Choose $h_{0}=1$. Some basic calculations give that $h_{0}<\frac{\Lambda}{2}=\frac{\pi}{2} \sqrt{\frac{1}{k_{2}-m_{2}}} \approx 1.61$. Furthermore, Theorem 4.5 shows that if $h_{0}<\frac{\Lambda}{2}$ then vanishing happens with small expanding capability $\mu$ and spreading happens with large expanding capability. From Figure 1a, we observe that the smaller the expanding capability $\mu$ is, the slower the free boundaries $x=g(t)$ and $x=h(t)$ increase, and $Z(t, x)$ decays to zero quickly. The numeric result shows that the top predator $Z(t, x)$ will eventually go to extinction, agreeing with Theorem 4.5. However, in Figure 1b, the larger the expanding capability $\mu$ is, the faster the free boundaries $x=g(t)$ and $x=h(t)$ increase, and $Z(t, x)$ stabilizes to a positive solution $Z_{3}^{*}$. This means that the top predator $Z(t, x)$ will eventually survive in the new environment. The numerical simulations illustrate the corresponding ecological explanation as follows. If the initial habitat $h_{0}$ is less than expanding barrier $\frac{\Lambda}{2}$, then the expanding capability $\mu$ dramatically affect the top predator $Z(t, x)$ whether spreading or vanishing occurs.

Example 5.2. Take $h_{0}=2>\frac{\Lambda}{2}$. Comparing with Figure 1a, we can see that in Figure 2 the free boundaries $x=g(t)$ and $x=h(t)$ increase fast, and $Z(t, x)$ stabilizes to a positive solution $Z_{3}^{*}$. It clearly shows that the top 
predator $Z(t, x)$ successfully spreads to the entire space as time goes to infinity. The corresponding ecological explanation depict that when the initial habitat $h_{0}$ is more than expanding barrier $\frac{\Lambda}{2}$, the top predator $Z(t, x)$ spreading always happens regardless of the expanding capability $\mu$. It turns out that Remark 4.2 is valid.

\section{CONCLUSION AND DISCUSSION}

In this work, we have studied the dynamics of a three species ratio-dependent food chain model with diffusion and double free boundaries in one dimensional space, in which the free boundaries represent expanding fronts of top predator species. We are mainly concerned with the case of a weak predation rate for the prey species (i.e., $\left.c_{1}<1\right)$. It is assumed that the top predator initially occupies a finite region $\left[-h_{0}, h_{0}\right]$ and has a tendency to expand its territories. The dynamic behavior of (1.4) exhibits a spreading-vanishing dichotomy, i.e., as $t \rightarrow \infty$, either

(i) the top predator $Z$ successfully establishes itself in the new environment in the sense that $g(t) \rightarrow-\infty$ and $h(t) \rightarrow \infty$. Moreover, $N \rightarrow N_{3}^{*}, P \rightarrow P_{3}^{*}$ and $Z \rightarrow Z_{3}^{*}$ when $k_{2}>m_{2}, k_{1}>m_{1}+c_{2}$ and $c_{1}<1$ hold (see Thm. 3.8);

or

(ii) the top predator $Z$ spreads within a bounded area and vanishes eventually, i.e., $h(t)-g(t) \rightarrow h_{\infty}-g_{\infty} \leq$ $\pi \sqrt{\frac{1}{k_{2}-m_{2}}}$ and $\|Z(t, x)\|_{C([g(t), h(t)])} \rightarrow 0$ when $k_{1}>m_{1}, k_{2}>m_{2}$ and $c_{1}<1$ hold. Furthermore, $N \rightarrow N_{2}^{*}$ and $P \rightarrow P_{2}^{*}$ uniformly in any compact subset of $\mathbb{R}$ (see Thm. 3.5).

The phenomena look more realistic and may play an important role in the understanding of ecological complexity.

Moreover, we provide the criteria for spreading and vanishing as follows. If the initial occupying habitat $\left[-h_{0}, h_{0}\right]$ is beyond a critical size, i.e., $2 h_{0} \geq \pi \sqrt{\frac{1}{k_{2}-m_{2}}}$, then spreading always happens regardless of the initial population density $\left(\phi_{1}, \phi_{2}, \phi_{3}\right)$ (see Rem. 4.2). In contrast, if $2 h_{0}<\pi \sqrt{\frac{1}{k_{2}-m_{2}}}$, then whether spreading or vanishing occurs is determined by the initial population density $\left(\phi_{1}, \phi_{2}, \phi_{3}\right)$ and the coefficient $\mu$ in the Stefan condition (see Thm. 4.5). Furthermore, the numerical simulations confirm our theoretical analysis for the food chain model rigorously.

To the best of our knowledge, Hsu et al. [10] formulated the ratio-dependent food chain model and thought that $N$ as a plant species, $P$ as pest species and $Z$ as a species used to control the pest. The purpose of the work was to study its applications to biological control from a mathematical point of view. Subsequently, a growing number of papers on the model have been published (e.g. [9] and [18]). A notable problem is under what conditions, all three species coexist. That is, the introduction of a natural enemy $Z$ for pest $P$ helps the population level of prey $N$. The authors gave some suitable coefficients conditions to ensure that all three species coexist. However, for problem (1.4) which is closer to reality than (1.2), main conclusions tell us that in order to control the pest species we further should put natural enemy at the initial state at least in one of the following three ways: (i) expand the initial habitat of natural enemy; (ii) increase the expanding capability; (iii) augment the initial density of the natural enemy.

The results in the present paper may enrich biological control in ecosystems.

Acknowledgements. We are very grateful to the anonymous referee for his/her careful reading and valuable suggestions which have led to an improvement of the presentation of the paper.

\section{REFERENCES}

[1] P.A. Abrams and L.R. Ginzburg, The nature of predation: prey dependent, ratio dependent or neither? Trends Ecol. Evol. 15 (2000) 337-341.

[2] G. Bunting, Y.H. Du and K. Krakowski, Spreading speed revisited: analysis of a free boundary model. Netw. Heterog. Media 7 (2012) 583-603.

[3] J.-F. Cao, W.-T. Li and M. Zhao, A nonlocal diffusion model with free boundaries in spatial heterogeneous environment. J. Math. Anal. Appl. 449 (2017) 1015-1035. 
[4] D.L. DeAngelis, R.A. Goldstein and R.V. O’Neill, A model for tropic interaction. Ecol. 56 (1975) 881-892.

[5] Y.H. Du, Z.M. Guo and R. Peng, A diffusive logistic model with a free boundary in time-periodic environment. J. Funct. Anal. 265 (2013) 2089-2142.

[6] Y.H. Du and Z.G. Lin, Spreading-vanishing dichotomy in the diffusive logistic model with a free boundary. SIAM J. Math. Anal. 42 (2010) 377-405.

[7] Y.H. Du and B.D. Lou, Spreading and vanishing in nonlinear diffusion problems with free boundaries. J. Eur. Math. Soc. 17 (2015) 2673-2724.

[8] Y.H. Du, M.X. Wang and M.L. Zhou, Semi-wave and spreading speed for the diffusive competition model with a free boundary. J. Math. Pures Appl. 107 (2017) 253-287.

[9] R. Feng, Spatiotemporal complexity of a three-species ratio-dependent food chain model. Nonlin. Dynam. 76 (2014) $1661-1676$.

[10] S.-B. Hsu, T.-W. Hwang and Y. Kuang, A ratio-dependent food chain model and its applications to biological control. Math. Biosci. 181 (2003) 55-83.

[11] O.A. Ladyzenskaja, V.A. Solonnikov and N.N. Ural'ceva, Linear and Quasilinear Equations of Parabolic Type. Academic Press, New York, London (1968).

[12] X.W. Liu and B.D. Lou, Asymptotic behavior of solutions to diffusion problems with Robin and free boundary conditions. MMNP 8 (2013) 18-32.

[13] J.B. Liao, J.H. Chen, Z.X. Ying, D.E. Hiebeler and I. Nijs, An extended patch-dynamic framework for food chains in fragmented landscapes. Sci. Rep. 6 (2016) 33100.

[14] J.B. Liao, D. Bearup and B. Blasius, Diverse responses of species to landscape fragmentation in a simple food chain. J. Anim. Ecol. 86 (2017) 1169-1178.

[15] H. Monobe and C.-H. Wu, On a free boundary problem for a reaction-diffusion-advection logistic model in heterogeneous environment. J. Differ. Equ. 261 (2016) 6144-6177.

[16] J.D. Murray, Mathematical Biology I: An introduction, 3rd ed. Spring-Verlag, New York (2002).

[17] C.V. Pao, Dynamics of food-chain models with density-dependent diffusion and ratio-dependent reaction function. J. Math. Anal. Appl. 433 (2016) 355-374.

[18] R. Peng, J.P. Shi and M.X. Wang, Stationary pattern of a ratio-dependent food chain model with diffusion. SIAM J. Appl. Math. 67 (2007) 1479-1503.

[19] R. Peng and X.-Q. Zhao, The diffusive logistic model with a free boundary and seasonal succession. Discrete Contin. Dyn. Syst. 33 (2013) 2007-2031.

[20] M.X. Wang, Stationary patterns for a prey-predator model with prey-dependent and ratio-dependent functional responses and diffusion. Phys. D 196 (2004) 172-192.

[21] M.X. Wang, The diffusive logistic equation with a free boundary and sign-changing coefficient. J. Differ. Equ. 258 (2015) $1252-1266$.

[22] M.X. Wang. A diffusive logistic equation with a free boundary and sign-changing coefficient in time-periodic environment. $J$. Funct. Anal. 270 (2016) 483-508.

[23] M.X. Wang, Existence and uniqueness of solutions of free boundary problems in heterogeneous environments. Discrete Contin. Dyn. Syst. Ser. B 24 (2019) 415-421.

[24] M.X. Wang and Q.Y. Zhang, Dynamics for the diffusive Leslie-Gower model with double free boundaries. Discrete Contin. Dyn. Syst. 38 (2018) 2591-2607.

[25] M.X. Wang and Y. Zhang, Note on a two-species competition-diffusion model with two free boundaries. Nonlin. Anal. 159 (2017) 458-467.

[26] M.X. Wang and Y. Zhang, Dynamics for a diffusive prey-predator model with different free boundaries. J. Differ. Equ. 264 (2018) 3527-3558.

[27] M.X. Wang and J.F. Zhao, Free boundary problems for a Lotka-Volterra competition system. J. Dynam. Differ. Equ. 26 (2014) 655-672.

[28] M.X. Wang and J.F. Zhao, A free boundary problem for the predator-prey model with double free boundaries. J. Dynam. Differ. Equ. 29 (2017) 957-979.

[29] K. Wonlyul and A. Inkyung, Dynamics of a simple food chain model with a ratio-dependent functional response. Nonlin. Anal. Real World Appl. 12 (2011) 1670-1680.

[30] C.-H. Wu, The minimal habitat size for spreading in a weak competition system with two free boundaries. J. Differ. Equ. 259 (2015) 873-897.

[31] D.W. Zhang and B.X. Dai, A free boundary problem for the diffusive intraguild predation model with intraspecific competition. J. Math. Anal. Appl. 474 (2019) 381-412.

[32] D.W. Zhang and B.X. Dai, The diffusive intraguild predation model with intraspecific competition and double free boundaries. Appl. Anal. (2020) DOI: 10.1080/00036811.2020.1716971.

[33] Y. Zhang and M.X. Wang, A free boundary problem of the ratio-dependent prey-predator model. Appl. Anal. 94 (2015) $2147-2167$.

[34] J.F. Zhao and M.X. Wang, A free boundary problem of a predator-prey model with higher dimension and heterogeneous environment. Nonlin. Anal. Real World Appl. 16 (2014) 250-263. 